\title{
A KDM4A-PAF1-mediated epigenomic network is essential for acute myeloid leukemia cell self- renewal and survival
}

Matthew E. Massett', Laura Monaghan (10', Shaun Patterson', Niamh Mannion', Roderick P. Bunschoten², Alex Hoose², Sandra Marmiroli ${ }^{3}$, Robert M. J. Liskamp ${ }^{2}$, Heather G. Jørgensen (1) ${ }^{1}$, David Vetrie ${ }^{4}$, Alison M. Michie and Xu Huang (1) ${ }^{1}$

\begin{abstract}
Epigenomic dysregulation is a common pathological feature in human hematological malignancies. H3K9me3 emerges as an important epigenomic marker in acute myeloid leukemia (AML). Its associated methyltransferases, such as SETDB1, suppress AML leukemogenesis, whilst H3K9me3 demethylases KDM4C is required for mixed-lineage leukemia rearranged AML. However, the specific role and molecular mechanism of action of another member of the KDM4 family, KDM4A has not previously been clearly defined. In this study, we delineated and functionally validated the epigenomic network regulated by KDM4A. We show that selective loss of KDM4A is sufficient to induce apoptosis in a broad spectrum of human AML cells. This detrimental phenotype results from a global accumulation of H3K9me3 and H3K27me3 at KDM4A targeted genomic loci thereby causing downregulation of a KDM4A-PAF1 controlled transcriptional program essential for leukemogenesis, distinct from that of KDM4C. From this regulatory network, we further extracted a KDM4A-9 gene signature enriched with leukemia stem cell activity; the KDM4A-9 score alone or in combination with the known LSC17 score, effectively stratifies high-risk AML patients. Together, these results establish the essential and unique role of KDM4A for AML self-renewal and survival, supporting further investigation of KDM4A and its targets as a potential therapeutic vulnerability in AML.
\end{abstract}

\section{Introduction}

Epigenetic regulators are frequently mutated in acute myeloid leukemia (AML), leading to epigenomic alterations ${ }^{1}$. Inhibitors that target epigenetic modulators to rectify epigenomic abnormalities represent valid therapeutic strategies. Further understanding of how epigenetic dysregulation in AML contributes to leukemogenesis may uncover tractable therapeutic targets and biomarkers for AML patient treatment and/or prognostic evaluation.

Polymerase-associated factor 1 (PAF1), a core component of the PAF complex (PAFc) is essential in $\mathrm{AML}^{2-4}$.

\footnotetext{
Correspondence: Xu Huang (xu.huang@glasgow.ac.uk)

${ }^{1}$ Paul O'Gorman Leukaemia Research Centre, Institute of Cancer Sciences,

University of Glasgow, Glasgow, United Kingdom

${ }^{2}$ Medicinal Chemistry Department, Joseph Black Building, School of Chemistry,

University of Glasgow, Glasgow, United Kingdom

Full list of author information is available at the end of the article

Edited by M. Diederich
}

PAFc mediated recruitment of the H3K9 methyltransferases, such as SETDB1 antagonizes Mixed-Lineage Leukemia (MLL) signaling, and inhibition of SETB1 promotes AML cell proliferation ${ }^{5,6}$.

Primary AML blasts from patients with poor prognosis feature global H3K9me3 hypomethylation ${ }^{7}$ positing an oncogenic role for H3K9me3 demethylases in AML. Cheung et al. identified an H3K9me3 demethylase, KDM4C as a cofactor of the PRMT1 complex in MLL rearranged (MLLr) and MOZ-TIF2 $\mathrm{AML}^{8}$. Simultaneous knockout (KO) of all three members of the $\mathrm{Kdm} 4$ family $(k d m 4 a / b / c)$ in mice attenuates MLL-AF9 $\mathrm{AML}^{9}$, indicating roles for the $\mathrm{Kdm} 4$ family in murine myeloid leukemia. However, the therapeutic benefit of targeting the KDM4 family in human AML is not well understood.

Our previous lentiviral knockdown (KD) screen targeting epigenetic regulators in 12 human AML cell lines

\section{(c) The Author(s) 2021}

(c) (i) Open Access This article is licensed under a Creative Commons Attribution 4.0 International License, which permits use, sharing, adaptation, distribution and reproduction cc) in any medium or format, as long as you give appropriate credit to the original author(s) and the source, provide a link to the Creative Commons license, and indicate if changes were made. The images or other third party material in this article are included in the article's Creative Commons license, unless indicated otherwise in a credit line to the material. If material is not included in the article's Creative Commons license and your intended use is not permitted by statutory regulation or exceeds the permitted use, you will need to obtain permission directly from the copyright holder. To view a copy of this license, visit http://creativecommons.org/licenses/by/4.0/. 
representing several AML subgroups found that KMD4A KD leads to significant suppression of leukemia cell proliferation ${ }^{10}$. KDM4A has different roles in normal tissue development compared to other members of the KDM4 family; it is amplified/overexpressed in various malignancies including AML (Fig. S1A) and correlates with poor outcome in ovarian cancer ${ }^{11,12}$. Herein we demonstrate that KDM4A KD induces AML apoptosis by a unique mechanism to $\mathrm{KDM} 4 \mathrm{C}$ in myeloid leukemia. Apoptosis results from a global accumulation of H3K9me3 and H3K27me3 at KDM4A genomic loci thereby causing downregulation of a KDM4A-PAF1mediated oncogenic program, including a 9-gene signature enriched with leukemia stem cell (LSC) activity, which can stratify high-risk patients. These findings support an essential and unique role of KDM4A for AML cell self-renewal and survival.

\section{Materials and methods}

Reagents, plasmids, and virus manufacture

Puromycin, IOX $1^{\text {dev }}$, and lentiviral constructs for $\mathrm{KD}$ experiments (Supplemental Table) were purchased from Sigma-Aldrich (St. Louis, MO, USA). IOX1 was from Tocris (4464). pLenti-HA-KDM4A wt and mut (H188A/ E190A) were a gift from Dr. Gary Spencer (CRUK Manchester Institute). Lentiviral and retroviral supernatants were prepared, and leukemic human and murine cells transduced with viral particles as previously described ${ }^{10}$. A list of antibodies used in flow cytometry and Western blot/immunoprecipitation and immunofluorescence staining is in the Supplemental Table.

\section{Culture of cell lines and primary cells}

Leukemia cell lines were from DMSZ (Braunschweig, Germany) and grown in RPMI (10\% FBS and $2 \mathrm{mM} \mathrm{L-}$ Glutamine) at $37^{\circ} \mathrm{C}$ in $5 \% \mathrm{CO}_{2}$. They were recently authenticated and tested for mycoplasma contamination. Murine and human primary AML and normal BM samples were cultured in serum-free media (SFM) (H5100, Stem Cell Technologies, UK) supplemented with appropriate cytokines as described ${ }^{13}$. Murine MLL-AF9 AML cells were leukemic BM cells extracted from a mice cohort with MLL-AF9 AML established by Somervaille et al. ${ }^{14}$, and cultured in the conditional medium with mIL-3 $(100 \mathrm{ng} / \mathrm{ml})$. Cytokines purchased from PeproTech (London, UK).

\section{Cell proliferation, apoptosis, and cell cycle analysis}

Cell viability was measured by cell count using trypan blue dye (Sigma, T8154)/hemocytometer or resazurin (Alamarblue dye, Sigma) with the Envision Fluorescent Reader (Perkin Elmer). Apoptosis was assessed using Annexin-V/Dead Cell Apoptosis Kit (V13241, ThermoFisher) and cell cycle analysis with PI/RNase Staining solution (F10797, ThermoFisher) as per manufactures' instructions. Data were acquired using an LSRII flow cytometer and an Aria III flow cytometer (BD Biosciences, UK) and analyzed using FlowJo software (Tree Star Inc., USA).

\section{Protein extraction and Western blot}

Cell pellets were suspended in fresh lysis buffer $(50 \mathrm{mM}$ Tris- $\mathrm{HCl}, \mathrm{pH} 7.4,150 \mathrm{mM} \mathrm{NaCl}, 1 \%$ Triton X-100, 0.5\% Sodium Deoxycholate, $0.5 \%$ SDS, $0.1 \% \beta$-mercaptoethanol, $10 \mathrm{mM}$ Sodium butyrate, $10 \mathrm{mM}$ nicotinamide, $1 \mathrm{mM}$ PMSF, $1 \times$ phosphatase inhibitor, $1 \times$ protease inhibitor) at $30 \mu \mathrm{l}$ per $10^{6}$ cells and lysed on ice. Protein Lysates were quantified using Pierce Coomassie Plus Bradford and ran in NuPAGE ${ }^{\mathrm{TM}} 4-12 \%$ Bis-Tris Protein Gels (Invitrogen) as per products' instructions. Only one loading control is used when Western blot analyses with multiple antibodies have been done sequentially in the same membrane.

\section{Immunofluorescence (IF) staining}

$6 \times 10^{4}$ cells per condition were incubated on poly-Llysine coated Hendley-Essex 12 well glass microscope slides for $1 \mathrm{~h}$ before being fixed in $4 \%$ formaldehyde in PBS. The cells were permeabilized in $0.5 \%$ Triton-X-100 PBS followed by $2 \mathrm{~h}$ of blocking in 5\% BSA, $0.2 \%$ Triton$\mathrm{X}-100$ TBS. Primary antibody was applied overnight in a humidified chamber at $4{ }^{\circ} \mathrm{C}$. Appropriate secondary antibody (1:500 dilution) was applied for further $1 \mathrm{~h}$ incubation at room temperature after removal of a primary antibody using PBS $0.1 \%$ Tween 20. Antifade mountant with DAPI reagent (Thermo Fisher \#P36962) was used to seal each sample and images were captured on the Zeiss Axioimager M1 Epifluorescence and Brightfield Microscope. CellProfiler v2.2.0 image analysis software (CellProfiler) was used to quantify IF signals.

\section{Colony-forming cell assay}

Colony-forming cell (CFC) assay for murine cells was performed by plating 1000 cells on methylcellulose (MethoCult M3434, Stem Cell Technologies). Colony Assay for human $\mathrm{CD} 34^{+}$hematopoietic stem and progenitor cells (HSPC) and AML patient cells were performed by plating 10000 cells and 3000 cells, respectively, on methylcellulose (MethoCult H4434, Stem Cell Technologies). CFU-GM (Granulocyte/Macrophage), M(Macrophage), and $E$ (Erythroid) colonies were enumerated 10 days after seeding.

\section{Murine transplantation experiments}

Mice experiments were approved by the local animal ethics board and performed under a project license issued by the United Kingdom Home Office, in keeping with the Home Office Animal Scientific Procedures Act, 1986. Non-obese diabetic. Cg-Prkdc scid Il2rgtm1Wjl/SzJ (NSG) mice were 
purchased from Jackson Laboratories (Bar Harbor, ME, USA) for transplantation as previously described ${ }^{10}$. Primary AML patient samples for xenograft transplantation were unfractioned primary blasts from our and Manchester biobank collections. Control or KDM4A KD human AML THP1 cells or primary AML patient blasts were FACS sorted $40 \mathrm{~h}$ following lentiviral infection and immediately transplanted into sub-lethally irradiated (450 cGy) NSG mice of 6-8-week-old, mixed-sex $\left(10,000 \mathrm{THP} 1\right.$ cells or $10^{\wedge} 6$ primary AML cells) via tail vein injection.

\section{RNA isolation, quantitative PCR, RNA-seq, and ChIP-seq}

RNA was extracted using QIAshredder ${ }^{\mathrm{TM}}$ columns and RNeasy Plus Microkit ${ }^{\mathrm{TM}}$ (Qiagen). RNA-seq libraries were produced using the TruSeq ${ }^{\circledR}$ stranded mRNA kit (Illumina) and sequenced on the Illumina NextSeq ${ }^{\mathrm{TM}} 500$ platform. For ChIP-seq, DNA was purified using Diagenode's iPure kitv2 and libraries made using the TruSeq ChIP Library Preparation Kit according to the manufacturer's instructions. For QPCR, reverse transcription was carried out using Invitrogen SuperScript III FirstStrand Synthesis kit. A SYBR ${ }^{\circledR}$ green-based fluorescent system was used to quantify dsDNA using the Applied Biosystems 7900 HT Fast Real-Time PCR system. Each qPCR plate included technical triplicates of each specific target alongside two housekeeping genes (GAPDH and BActin). Delta-Delta CT method was used for analysis of gene expression against control. RNA-seq and ChIP-seq reads were mapped to the hg19 human genome or $\mathrm{mm} 9$. Transcript abundances were calculated in transcripts per million (TPM) using Kallisto ${ }^{15}$. SICER was used for peak calling on default settings. Both data files are available in the Gene Expression Omnibus (GEO): GSE125376.

\section{Gene signature construction}

The KDM4A-9 signature score is calculated as shown below using the least absolute shrinkage and selection operator (LASSO) linear regression ${ }^{16,17}$.

$$
K D M 4 A-9 \text { signature score }=\sum_{n=1}^{9} x_{i} r_{i}
$$

Where $x_{\mathrm{i}}$ and $r_{\mathrm{i}}$ denote the gene expression and coefficient of the $i$ th gene (out of the total 9 genes) in the signature, respectively. Specifically, KDM4A-9 score $=(T P M 2 \times 0.097292)+(C D 82 \times 0.038719)+(S L C 29 A 2 \times$ $0.167487)+(I N F 2 \times 0.150025)+(S T A R \times-0.13354)+$ $(A C P 6 \times 0.04633)+(I F I 6 \times 0.015753)+(M R O H 6 \times$ $0.086738)+(G S D M D \times 0.008289)$.

\section{Correlating the $K D M 4 A-9$ signature and LSC activity}

LSC enrichment classification and raw expression data from AML samples (GSE76008) ${ }^{17}$ were analyzed using the lumi 2.36.0 package. Intensity values were normalized by
Robust spline normalization. The diagnostic capability of each gene signature to predict LSC activity across AML samples (GSE76008) was assessed by ROC (Receiver operating characteristic) curve analysis. The Youden index was used to identify the optimal cut-off value.

\section{Construction of KDM4A-9 and LSC17 combined score}

Min-Max scaling of the KDM4A-9 and LSC17 scores was performed prior to the linear summation of $K D M 4 A$ 9 and $L S C 17$ scores for each patient to generate a combined KDM4A-9/LSC17 score.

$$
X_{\text {new }}=\frac{X_{i}-\min (X)}{\max (X)-\min (X)}
$$

\section{Network construction and visualization}

To visualize the connections between the LSC17 and KDM4A-9 signature genes, as well as KDM4A and PAF1 in AML patients, a filtered edge list $(\mathrm{TOM} \geq 0.05)^{18}$ was constructed. An undirected network graph was generated using the graph.edgelist function from the igraph 1.2.5 package.

\section{Motif analysis}

Motif enrichment analysis of $400 \mathrm{bp}$ length DNA sequences centered over the TSS of KDM4A or PAF1 bound $(\mathrm{FDR} \leq 0.01)$ and $\mathrm{DE}$ (padj $\leq 0.05, \mathrm{DE} \geq 0.5$ or $\leq-0.5)$ genes was performed using MEME-ChIP (http:// meme-suite.org/tools/meme-chip).

\section{Statistical analysis}

Normally distributed groups were compared using a two-tailed student $t$-test unless stated otherwise. Survival probabilities estimated by Kaplan-Meier method. For RNA-seq counts, a pseudo-count of 1 was added prior to $\log _{2}$-transformation. Statistical significance of differential gene expression was assessed by Welch's $t$-test unless otherwise stated. For RNA-seq, differential expression analysis was performed using the DESeq2 1.26.0 R package. Statistics were calculated using R-3.6.1.

\section{Results}

KDM4A is required for the survival of human and murine AML cells

$K D M 4 A$ expression is unique (Fig. S1B) being highly enriched in AML-LSC ${ }^{+}$populations (Fig. S1C), suggesting that KDM4A is important for LSC, which are negatively correlated with AML patient survival. We performed lentivirus shRNA KD of $K D M 4 A, K D M 4 B$, and $K D M 4 C$ in human AML MLL-AF9-driven THP1 cells to confirm its essential role. KDM4A KD THP1 cells exhibited the greatest decrease in cell proliferation compared to nontargeting cells (NTC) (Fig. 1A-C). Consistent with 


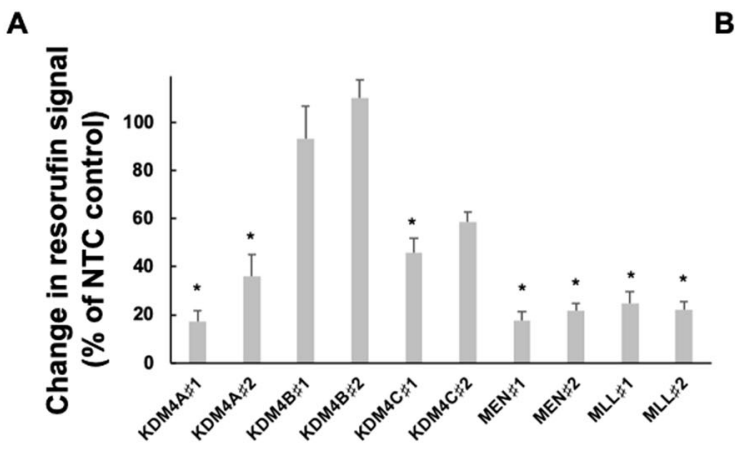

C

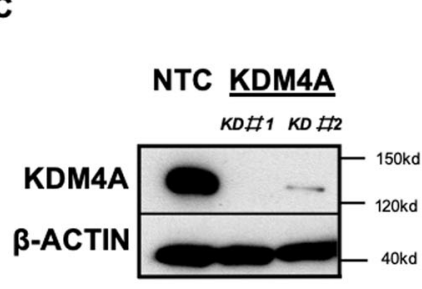

F

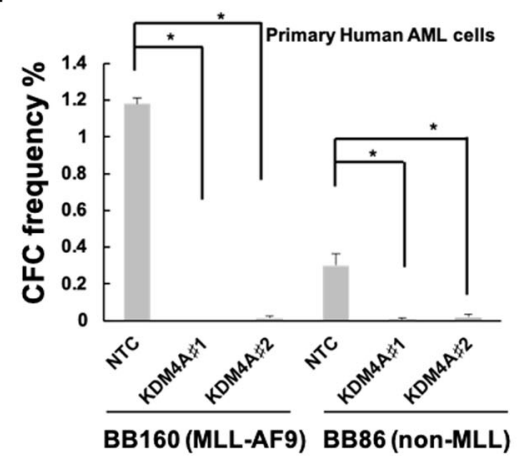

I

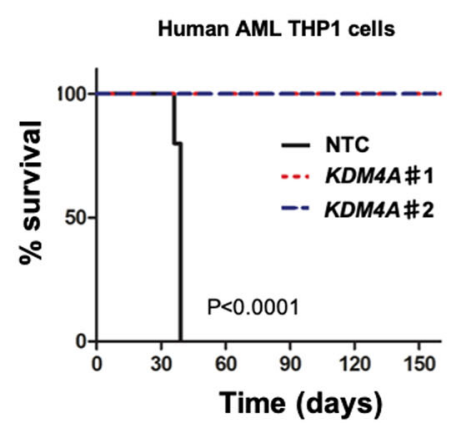

D

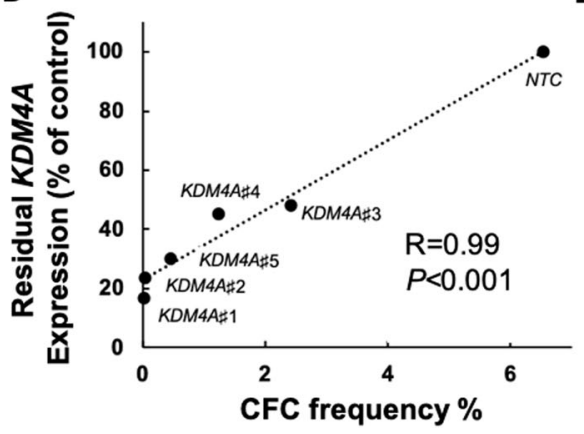

E

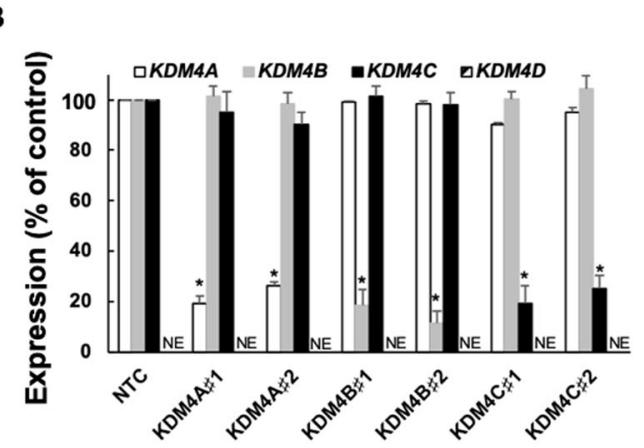

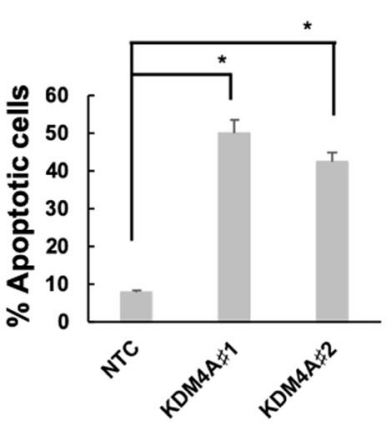

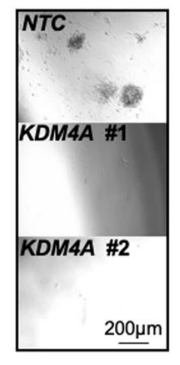

$200 \mu \mathrm{m}$

H

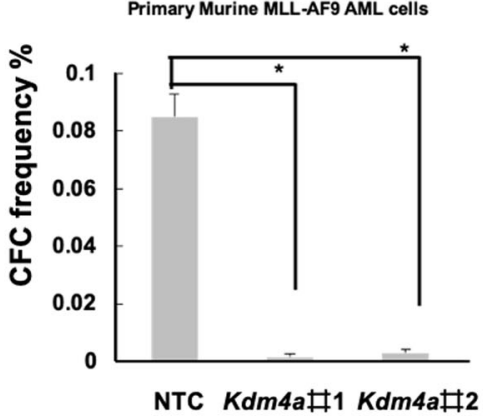

K
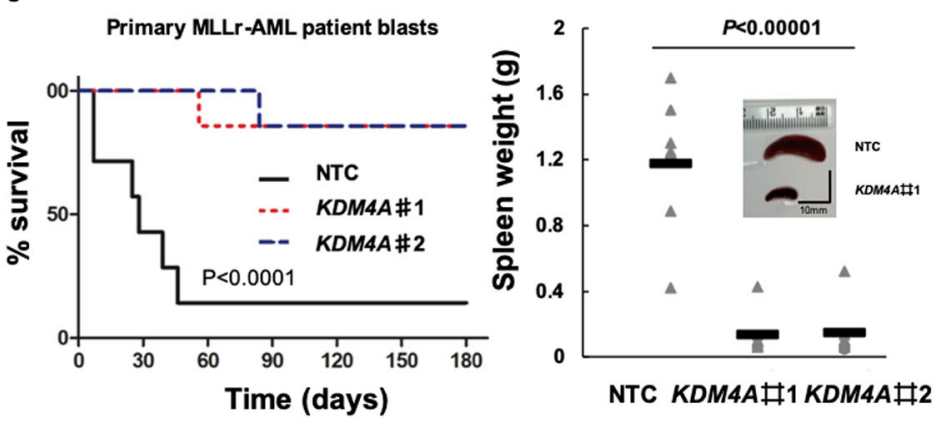
(see figure on previous page)

Fig. 1 KDM4A is required for the functional potential of human and murine AML cells. A-E Human THP1 AML cells were transduced with lentiviruses targeting KDM4A or other KDM4 family members for $\mathrm{KD}$ ( $\# 1$ and $\sharp 2$ represent individual distinct lentiviruses targeting genes for $\mathrm{KD}$ as indicated), or a non-targeting control (NTC), using KD of MLL and MEN1, as positive controls, which are essential genes for AML cell proliferation. All bar charts show mean \pm s.e.m. A Resorufin signal after 4 days of individual KDM4 family member KD relative to NTC control cells $(n=3)$; ${ }^{*} p<0.01$ for comparison of each KD versus NTC. B Expression of KDM4A/B/C/D in indicated KD cells relative to NTC control cells $(n=3) ;{ }^{*} p<0.001$. C Representative immunoblot showing KDM4A KD in THP1 cells $(n=3)$. D Scatter plot shows the correlation of KDM4A KD with inhibition of frequency of colonyforming cells (CFC) enumerated following 10 days in semisolid culture $(n=3)$, as determined by QPCR; ${ }^{*} p<0.001$. E Percentage of apoptotic cells determined by Annexin $\mathrm{V}^{+} / 7 \mathrm{AAD}^{+/-}$staining on day 4 of liquid culture after puromycin selection $(n=3)$; ${ }^{*}<0.001$. F-G The indicated primary unfractioned patient blasts were transduced with lentiviruses targeting KDM4A for KD, or an NTC. Primary AML cells used include BB160, containing $t$ (9;11) (MLL-AF9) chromosomal translocation and BB86 (normal cytogenetics, non-MLL) (BB number is the Manchester Cancer Research Centre Biobank sample identifier). All bar charts show mean \pm s.e.m. F CFC frequencies of primary human AML blasts $(n=3)$ following lentivirus infection, puromycin selection, and initiation of $K D M 4 A \mathrm{KD} ;{ }^{*} p<0.0001$. G Representative images from $\mathbf{F}$. H CFC frequencies of primary murine MLL-AF9 AML cells following KDM4A depletion ( $n=3$ ); ${ }^{*} p<0.0001$. I Survival curves of NSG mice transplanted with 10,000 KDM4A KD or NTC THP1 cells ( $n=5$ per cohort); $p$ by logrank test. J Survival curves of NSG mice transplanted with $10^{6} \mathrm{KDM} 4 \mathrm{~A}$ KD or NTC primary AML cells (BB160, $n=7$ per cohort); $p$ by log-rank test. $\mathbf{K}$ Spleen weights of mice from $\mathbf{J}$ with a representative image of the spleen. $p$ by one-way ANOVA, $F=34.13045$.

previous work, lentiviral KD of $K D M 4 C$ had an inhibitory effect on cell proliferation ${ }^{8}$ (Fig. 1A). CFC potential was positively correlated with the $K D M 4 A$ in a dosedependent manner when five KDM4A KD shRNA targeting constructs were compared (Fig. 1D). KDM4A KD induced apoptosis (Fig. 1E and S1D) rather than cell cycle arrest (Fig. S1E). These results were further confirmed in primary patient blasts (Fig. $1 \mathrm{~F}$ and G) and murine AML cells (Fig. $1 \mathrm{H}$ ). Importantly, we determined the impact of $K D M 4 A \mathrm{KD}$ on AML initiation in vivo by transplanting KDM4A KD THP1 cells (Fig. 1I) or primary AML cells (Fig. 1J-K and S1F-S1H) into recipient NSG mice. Control cells induced short-latency disease with splenomegaly (Fig. 1K). Loss of KDM4A significantly prolonged overall survival (OS) of mice with only one mouse succumbing to leukemia over the follow-up period by either KDM4A\#1 KD or KDM4A\#2 KD (Fig. 1J-K and S1F$\mathrm{S} 1 \mathrm{H})$. Together, these data demonstrate a specific and essential role for KDM4A in AML cell survival.

\section{Targeting KDM4A's demethylase activity inhibits AML cell proliferation}

Functional rescue experiments determined that the demethylase activity of KDM4A is required for AML. Forced-expression of wild-type human KDM4A rescued the clonogenic activity of AML cells transduced with $k d m 4 a \mathrm{KD}$ virus (Fig. $2 \mathrm{~A}$ and $\mathrm{B}$ ). This rescue phenotype was not observed by a catalytically inactive mutant of KDM4A (KDM4A $\left.{ }^{\mathrm{H188A} / \mathrm{E} 190 \mathrm{~A}}\right)^{19,20}$ (Fig. 2A and B) in murine MLL-AF9 cells. Next, we assessed KDM4A substrates H3K9me3 and H3K36me3 in KDM4A KD THP1 cells. There was an increase in H3K9me3 shown by immunoblotting (Fig. 2C and D) and immunofluorescent staining (Figs. S2A-S2B). No significant changes in H3K36me3 were observed (Fig. 2C-D; S2A-S2B), suggesting $\mathrm{H} 3 \mathrm{~K} 9 \mathrm{me} 3$ is the primary target of KDM4A in
THP1 cells. In addition, there was a marked elevation of H3K27me3 in KDM4A KD THP1 cells (Fig. 2C-D; S2A$\mathrm{S} 2 \mathrm{~B}$ ) and two further KDM4A KD human MLLr-AML cell lines (Figs. S2C-S2D).

We next investigated whether KDM4A is dispensable for normal hematopoiesis. Our data showed no significant loss of colonies in $k d m 4 a \mathrm{KD}$ normal murine BM c-kit ${ }^{+}$cells in CFC assays (Fig. 2E and F; S2E). Similarly, reduced levels of $K D M 4 A$ in human CD34 ${ }^{+}$HSPCs from healthy donors are tolerated (Fig. 2G and H; S2F) with fewer colonies due to a reduction of CFU-GM in KDM4A KD \#1 cells. Although a KDM4A specific inhibitor is lacking, there are several panKDM4 inhibitors available including $\mathrm{IOX}^{21}$ and IOX derivatives $\left(\mathrm{IOX} 1^{\mathrm{dev}}\right)^{22,23}$. These displayed significant inhibitions of cell proliferation in THP1 cells and primary AML patient blasts, inducing differentiation and apoptosis (Figs. S3A-S3G) with minimum effect on normal human $\mathrm{CD}^{+}{ }^{+}$BM HSPCs (Fig. S3E). These phenotypes were accompanied by an increased level of H3K9me3 and H3K27me3 (Fig. S3H), suggesting the anti-leukemic effect was due to KDM4A inhibition.

\section{PAF1 identified as a KDM4A co-regulator is required for human AML cell survival}

To determine the impact of KDM4A on global gene expression, we compared the transcriptome of KDM4A KD THP1 cells with NTC control by RNA-seq. 3375 differentially expressed (DE) genes were significantly deregulated following depletion of $K D M 4 A$ ( $\log _{2}$ fold change (FC) $\geq 0.5$ or $\leq-0.5$; adj. $p \leq 0.05$; Fig. 3A; Supplemental file). $67 \%$ (2274 out of 3375 ) were direct targets of KDM4A; ChIP-seq revealed that KDM4A bound at their TSS (Supplemental file). 61\% (1387 out of 2274) of putative KDM4A direct target genes were downregulated associated with the enrichment of transcriptional repressive marks at $\mathrm{H} 3 \mathrm{~K} 9 \mathrm{me} 3^{24}$ and $\mathrm{H} 3 \mathrm{~K} 27 \mathrm{me} 3^{25}$ (Fig. 3A). 


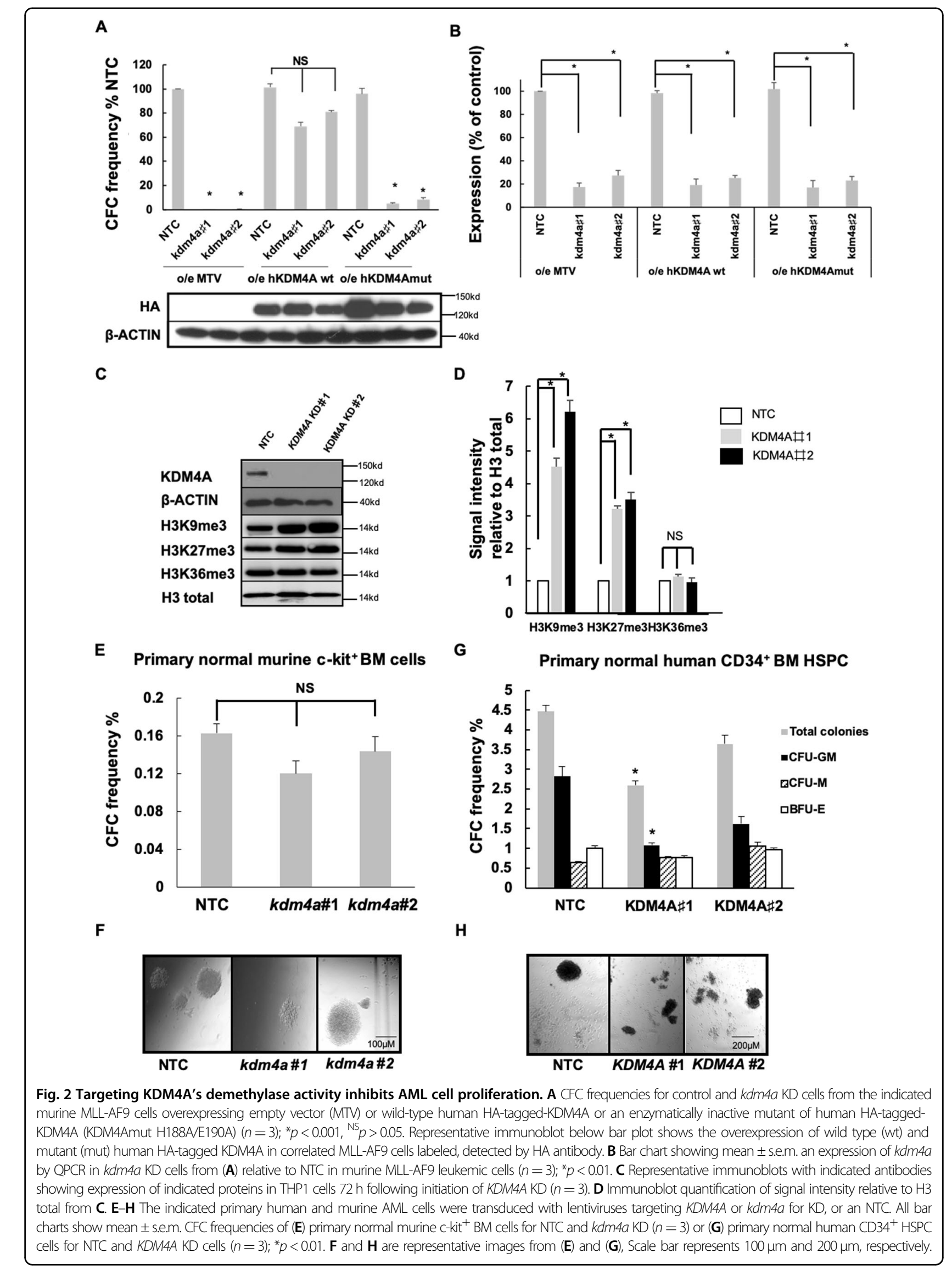

E Primary normal murine c-kit ${ }^{+} \mathrm{BM}$ cells

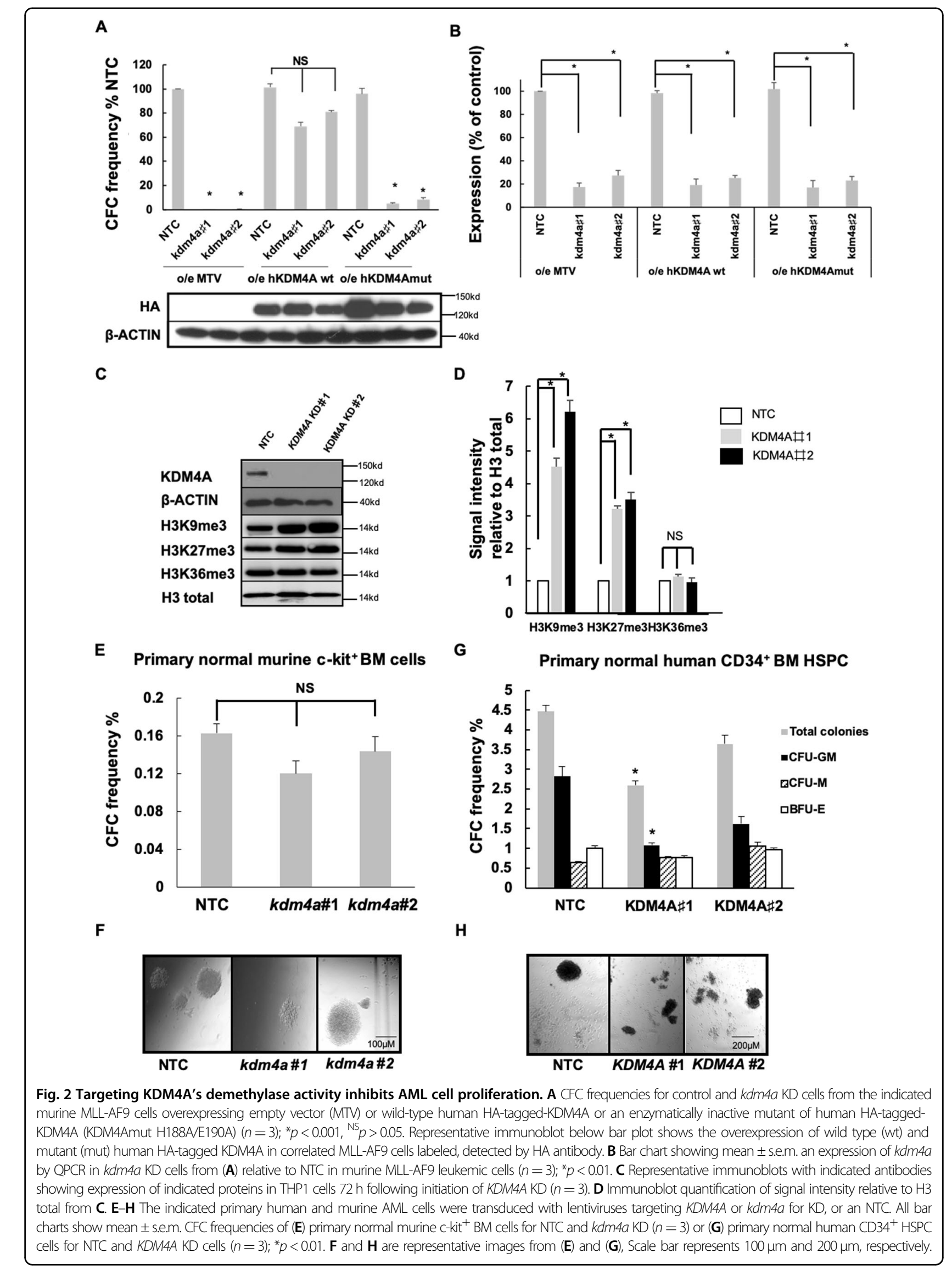


A

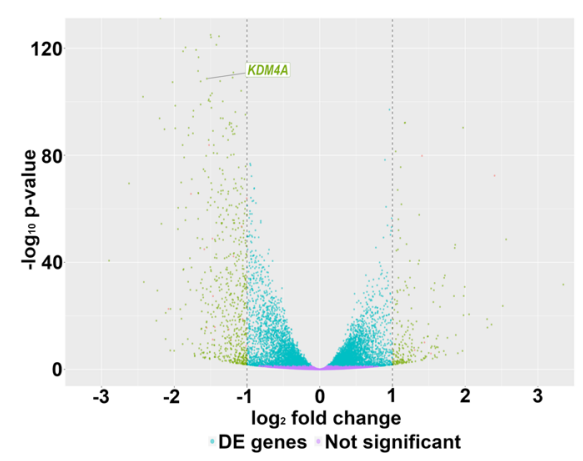

C

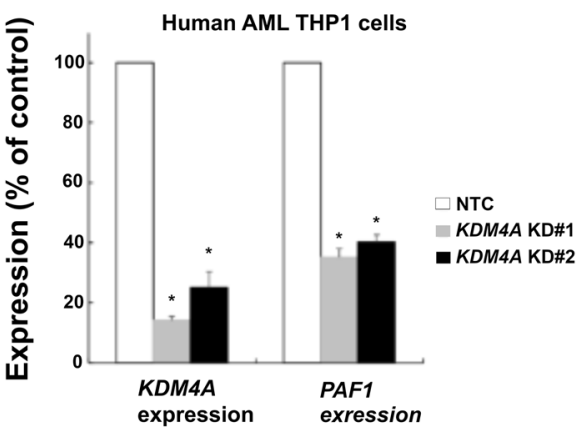

E

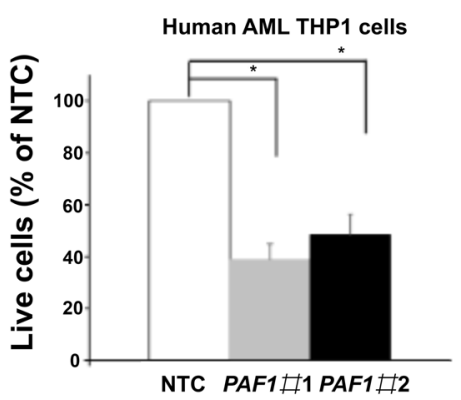

G

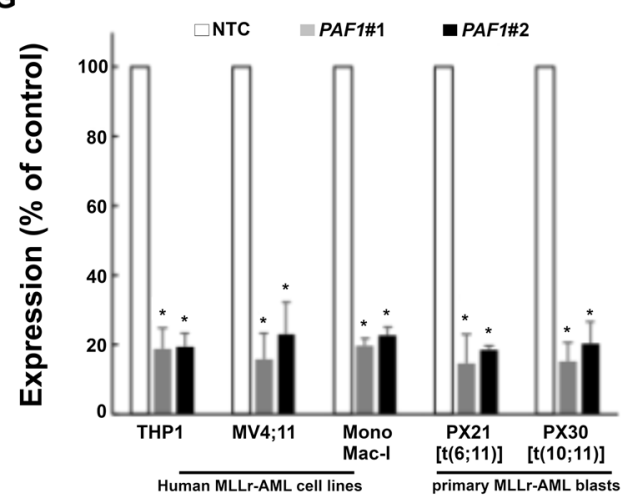

B

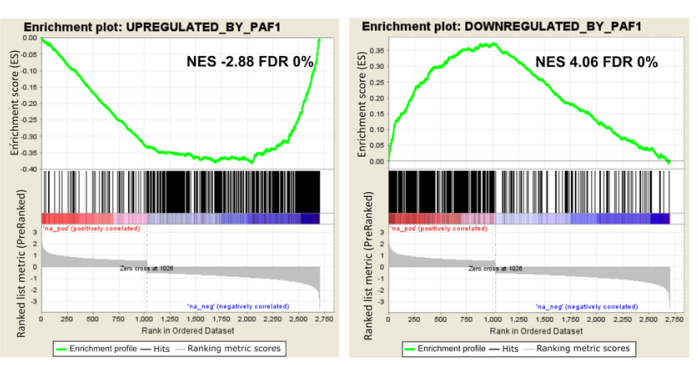

D

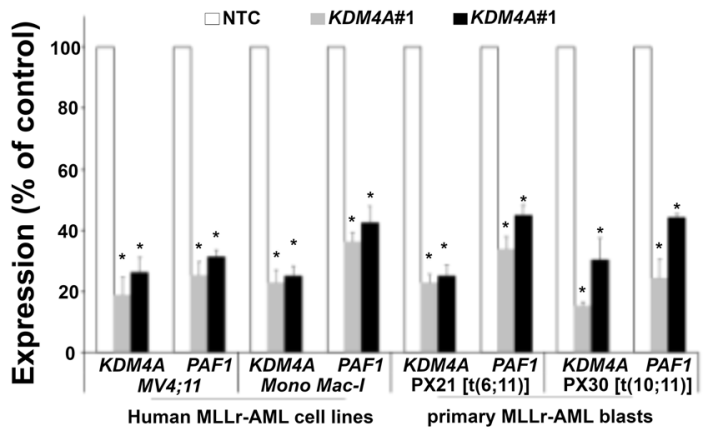

F

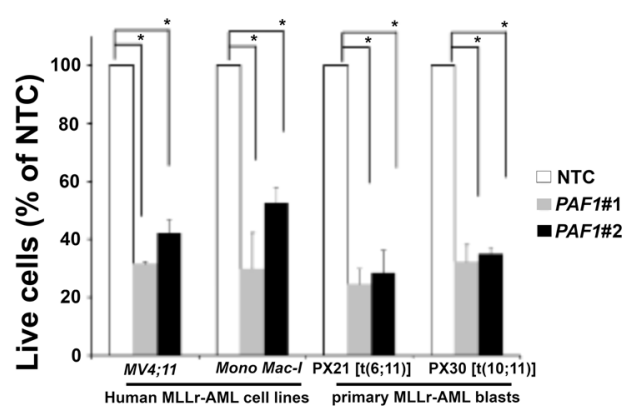

H

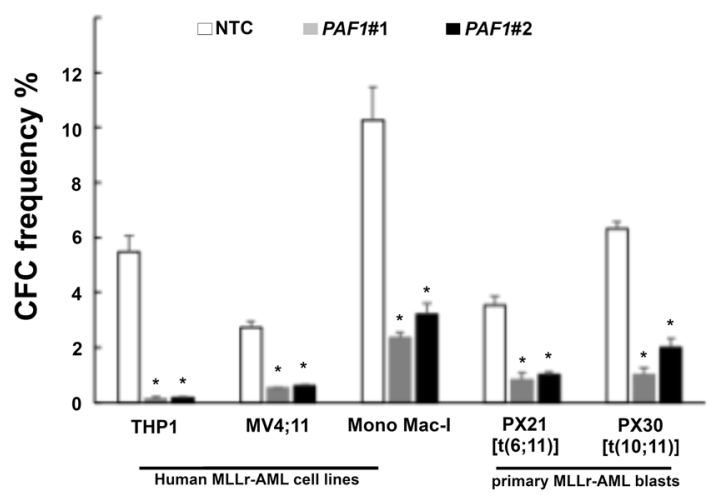

Fig. 3 (See legend on next page.) 
(see figure on previous page)

Fig. 3 PAF1 identified as a cofactor of KDM4A in MLLr-AML. A Volcano plot showing global changes in gene expression following the loss of KDM4A compared to NTC control THP1 cells as identified by RNAseq. The absolute number of upregulated or downregulated genes that are bound by KDM4A is indicated at the top right and left side of the plot, respectively (KDM4A bound: FDR $\leq 0.01$, gene expression: $\log _{2} F C \geq 0.5$ or $\leq-0.5$; adjusted (adj.p) $p \leq 0.05$ ). B GSEA show overlapping transcriptional consequences following the loss of KDM4A or PAF1 in THP1 cells. Specifically, genes repressed (left panel) or activated (right panel) are upregulated and downregulated, respectively, following the loss of KDM4A ${ }^{26}$. C-H THP1 cells and other indicated human AML cells were transduced with lentiviruses targeting KDM4A or PAF1 for KD, or an NTC. All bar charts show mean \pm s.e.m. C Bar chart showing relative expression of KDM4A and PAF1 by QPCR in comparison with NTC control cells following KDM4A KD using two different shRNA constructs $\sharp 1$ and $\sharp 2$ in THP1 cells $(n=3)(\mathbf{D})$ and the other indicated human AML cells and primary AML cells include PX21, containing $\mathrm{t}(6 ; 11)$ (MLL-AF6) chromosomal translocation and PX30 t(10;11)(MLL-AF10) (PX number is the Paul O'Gorman Leukaemia Research Centre Biobank sample identifier) $(n=3)(\mathbf{F}) ;{ }^{*} p<0.01$. E-F Percentage of live cell counts in comparison with NTC control (E) in THP1 cells 4 days following lentiviral infection $(n=3)$ and $(\mathbf{F})$ in the indicated human MLLr-AML cell lines and AML primary cells following KDM4A KD in comparison with NTC control cells $(n=3)(\mathbf{I}){ }^{*} p<0.01$. G Bar chart showing relative expression of PAF1 by QPCR in comparison with NTC control cells following PAF1 KD using two different shRNA constructs $\sharp 1$ and $\sharp 2$ in (E and $\mathbf{F})$. $\mathbf{H}$ Bar chart showing the loss of CFC frequencies of indicated human AML cell lines and primary patient samples $(n=3)$ following lentivirus infection, puromycin selection, and initiation of PAF1 KD; ${ }^{*} p<0.001$.

To provide insights into the survival pathways regulated by KDM4A, we performed gene-set enrichment analysis (GSEA) and revealed significant enrichment of genes regulated by $\mathrm{PAFc}^{26-28}$ (Fig. 3B). This is consistent with downregulation of $P A F 1$, following KDM4A KD at the transcript (Fig. $3 \mathrm{C}$ and D) and protein (Fig. S4A) level in human MLLr-AML cell lines, and primary patient blasts. PAF1 KD phenocopied KDM4A KD in MLLr-AML cells, inducing significant apoptosis (Fig. 3E-G; S4B) and loss of CFU potential (Fig. 3H). Together, these data suggest loss of KDM4A impairs PAF1 function to maintain leukemic cell survival, supporting PAF1 as an important cofactor of KDM4A in human AML.

\section{KDM4A-PAF1 maintains appropriate expression of the MLLr-fusion oncogenic program in MLLr-AML}

Our ChIP-seq data reveal substantial overlap amongst $\mathrm{PAFc}^{26}$, MLL-AF9 ${ }^{29}$, and KDM4A binding sites (Fig. 4AC). Specifically, KDM4A bound the PAF1 promoter region (Supplemental file), suggesting a direct regulatory mechanism. There is no enrichment of either histone methylation mark at non-KDM4A binding genomic loci (Fig. 4D and E), indicative of a human KDM4A-specific epigenomic profile. In marked contrast, there is a global gain of both H3K9me3 and H3K27me3 upon KDM4A KD in THP1 cells at KDM4A binding sites (Fig. 4F and G; $5 \mathrm{~A}$ ), including the genomic loci of PAF1 and its targets (Fig. 5B). These were validated by ChIP-QPCR in cell lines and primary patient blasts (Fig. $5 \mathrm{C}$ and D).

Furthermore, genes with significant expression changes following KDM4A silencing were also enriched in direct PAF1 target genes ${ }^{26}$ (Fig. 5E), suggesting a transcriptional network co-regulated by both KDM4A and PAF1. This notion is supported by the fact that KDM4A bound promoters share almost identical enrichment of transcription factor (TF) binding motifs as the ones bound by PAF1, including homeobox (HOX) TFs, such as TLX2 and DBX (Fig. 5F). Further GSEA analysis on the overlapped DE genes between KDM4A KD and PAF1 KD revealed a significant downregulation of MLLr fusion target genes ${ }^{30}$, as well as HOX family target genes ${ }^{31}$ including notably the pro-survival gene, BCL2, and marked upregulation of a mature hematopoiesis program $^{32}$ (Fig. 5G) and pro-apoptotic gene, BCL2L11 (BIM). Although the expression of HOXA9 itself was not affected by either KD, our data suggest KDM4A and PAF1 coregulate their downstream targets in a parallel manner.

\section{A core 9-gene signature downstream of $K D M 4 A$ strongly associated with LSC activity and clinical outcome}

Supporting the collaborative role of KDM4A-PAF1 in AML, KDM4A expression is highly associated with PAF1 expression in patient datasets (Fig. 6A and B); KDM4APAF1 expression can identify patients with inferior OS (Fig. 6C). KDM4A KD induced a significant reduction of cell proliferation in human AML cell lines representative of different subtypes (Fig. S5A), coupled with an increase in apoptosis and loss of CFC potential (Figs. S5B and $\mathrm{S} 5 \mathrm{C})$. This evidence suggests that KDM4A is required across AML.

These data led to our hypothesis that a core gene expression signature (GES) downstream of the KDM4APAF1 regulatory axis, is associated with AML patient outcomes compared with the known LSC score, $L S C 17^{17}$. For this, we utilized LASSO regression analysis ${ }^{16,17,33}$ on KDM4A regulated genes (KDM4A KD $\log _{2} \mathrm{FC} \geq 1$ or $\leq-1$; adj. $p \leq 0.05$; Supplemental file) and defined a $K D M 4 A-9$ score (Fig. 6D). High KDM4A-9 was highly associated with poor OS in AML cohorts (Fig. 6E-H) independent of age, cytogenetic risk score, and frequent mutation status. The robust prognostic value of KDM4A-9 indicates that the score may be related to the important biological activities of AML-LSCs. Indeed, KDM4A-9 correlates with the $L S C 17$ score of AML samples and over $75 \%$ of KDM4A-9 high score fractions are LSC+ (Fig. 7A), therefore $K D M 4 A-9$ is a strong predictive indicator of 
A

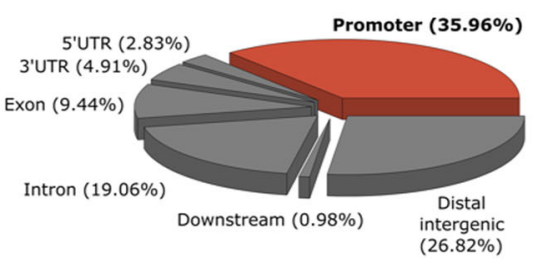

KDM4A binding genomic location

C

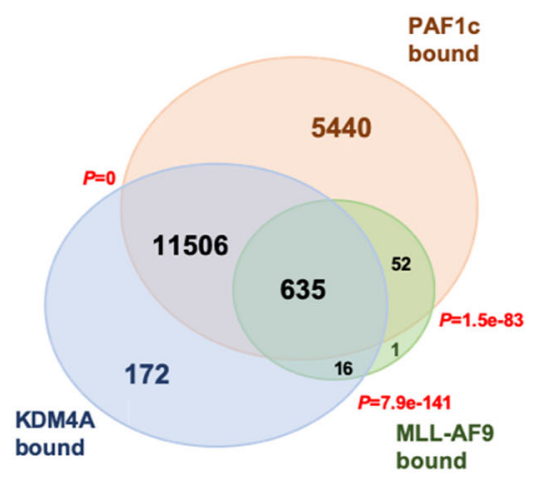

D

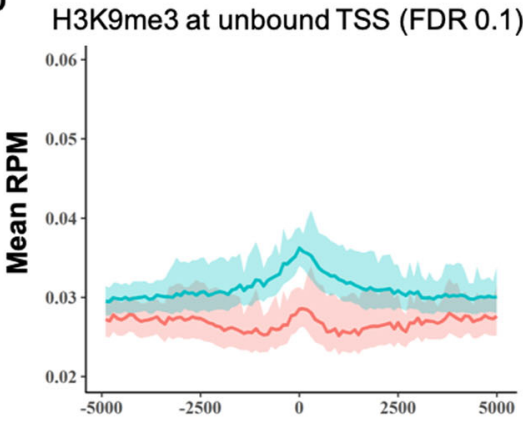

Distance (nucleotides) from TSS

F $\quad \mathrm{H} 3 \mathrm{~K} 9 \mathrm{me} 3$ at bound TSS (FDR 0.1)

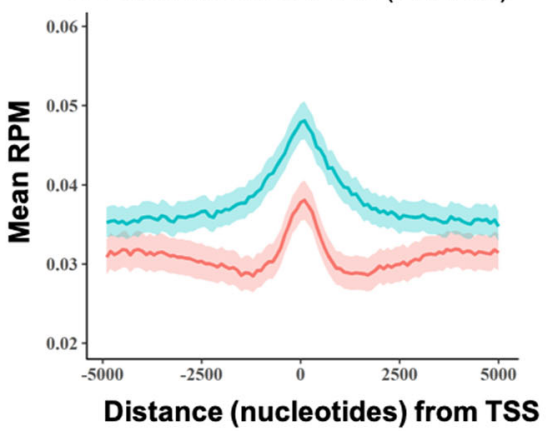

B

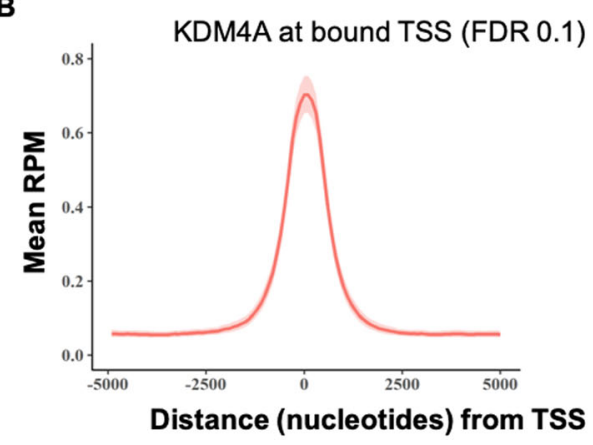

E

NTC

KDM4A甘1
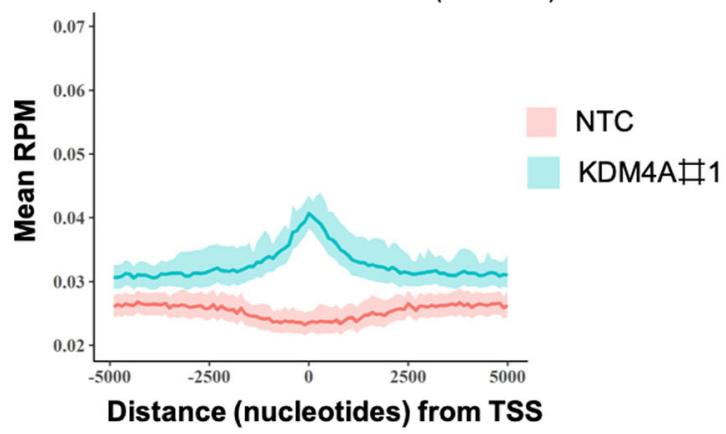

G

H3K27me3 at bound TSS (FDR 0.1)

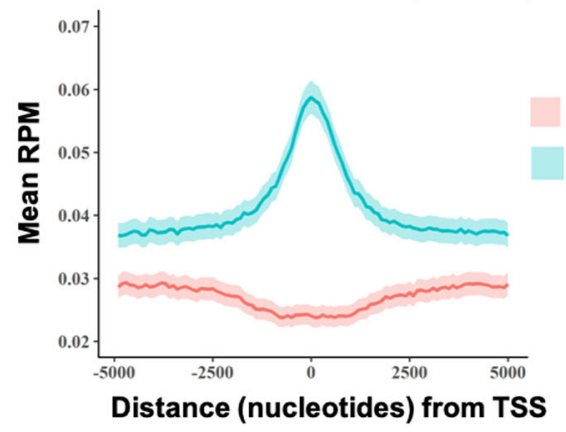

NTC

KDM4A\#1

Fig. 4 KDM4A-PAF1 co-regulates essential MLLr-fusion oncogenic transcriptional program. A Feature distribution of KDM4A ChIP-seq peaks in the THP1 cell genome. B Metagene plots showing a distinct peak in KDM4A normalized ChIP-seq signal in reads per million mapped reads (RPM) at transcription starting sites (TSS) in WT THP1 cells. C Venn diagram showing the overlap between binding sites of KDM4A, PAF1 ${ }^{26}$, and MLL-AF9 ${ }^{29}$ in THP1 cells as determined by ChIP-seq; $p$ by hypergeometric test. D-G Metagene plots showing enrichment of H3K9me3 (F) and H3K27me3 (G) at KDM4A bound TSS compared to unbound TSS (D and $\mathbf{E}$ ) following KDM4A KD by ChIP-seq. 
A

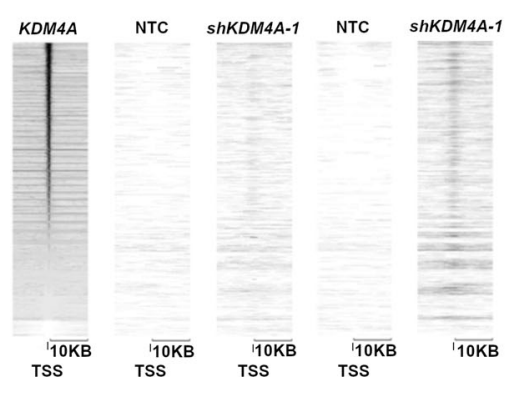

C

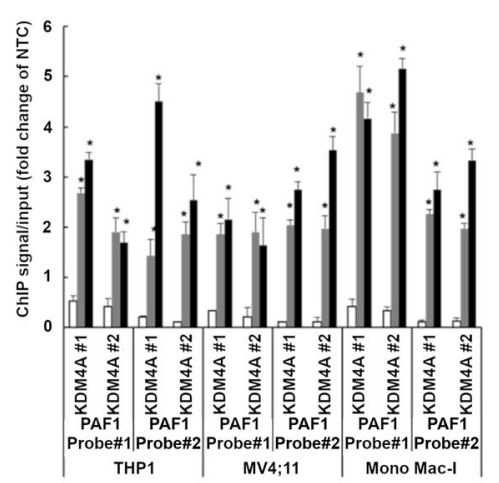

E

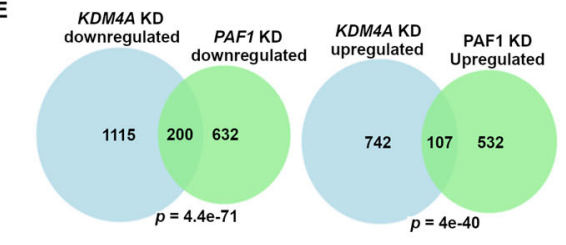

G
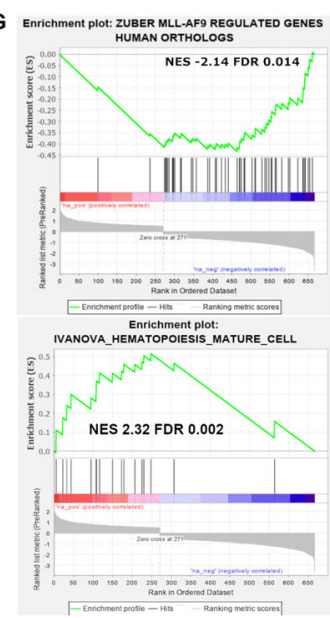

D
B
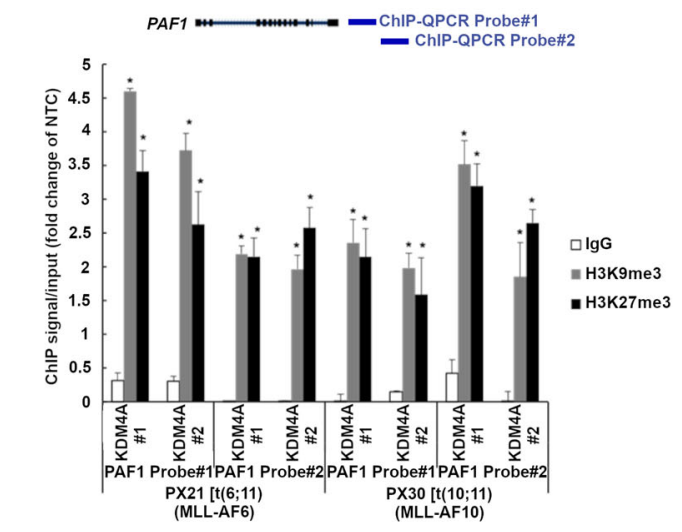

$\mathbf{F}$

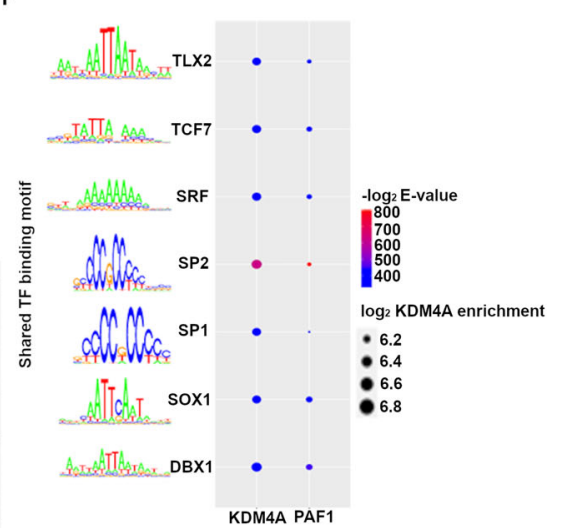

KDM4A PAF1

Fig. 5 (See legend on next page.) 
(see figure on previous page)

Fig. 5 KDM4A-PAF1 maintains appropriate expression of the MLLr-fusion oncogenic program in MLLr-AML. A Heatmap showing normalized ChIP-seq signal of H3K9me3 and H3K27me3 at TSS across all genes in KDM4A KD and NTC THP1 cells ordered by KDM4A enrichment. B Genomic snapshot demonstrates KDM4A occupancy at the PAF1 promoter region and enrichment of H3K9me3 and H3K27me3 signal throughout the PAF1 gene body and promoter upon KDM4A KD in comparison with NTC control in THP1 cells. Blue bars show the two individual probes used for ChIPQPCR in C and D. C-D H3K9me3 and H3K27me3 ChIP signal/input (fold change of NTC) in the indicated human MLLr-AML cell lines (C) and indicated primary AML samples including PX21 (MLL-AF6) and PX30 (MLL-FA10) (D) as determined by ChIP-QPCR following depletion of $K D M 4 A ;{ }^{*} p<0.001$. E Venn diagrams showing the overlap between directly bound downregulated and upregulated targets of KDM4A and the PAF1 $c^{24}$ in THP1 cells following knockdown of KDM4A and PAF1 as determined by ChIPseq; $p$ by hypergeometric test. $\mathbf{F}$ Motif significance and KDM4A log 2 enrichment at KDM4A or PAF1 regulated promoters ( $F D R \leq 0.01, D E \leq-0.5$, or $D E \geq 0.5$ ). Color represents motif significance within KDM4A and PAF1 regulated promoters. Size denotes the average $\log _{2}$ enrichment of KDM4A within each group of promoters that possess the respective transcription factor (TF) binding motif. Top five motifs detected in KDM4A or PAF1 regulated promoters sorted by statistical significance (E-value). G GSEA results showing significant overlap of KDM4A KD transcriptional consequences with downregulation of MLL-AF9 and HOXA9 targets and upregulation of a mature hematopoiesis program in THP1 cells, ${ }^{*} q<5 \%$.

AML-LSC activity (Fig. 7B). Interestingly, there is no overlap between these two gene signatures. A combined signature score (KDM4A-9/LSC17) achieves an optimal balance between specificity and sensitivity (Fig. 7C) overcoming the limitations of either score alone with an improved ability to predict survival over LSC17 (Fig. 7D and $\mathrm{E})$.

KDM4A binds at the promoter regions of KDM4A-9 genes, whereupon H3K9me3 and H3K27me3 are enriched after KDM4A KD (Fig. 8A; Supplemental file), suggesting direct regulation. In addition to the QPCR validation in human AML cell lines and primary AML blasts following KDM4A KD or PAF1 KD (Fig. 8B), we observed that the majority of KDM4A-9 genes show correlation with KDM4A and PAF1 expression in patient AML cohorts (Fig. 8C and D). Furthermore, weighted gene correlation network analysis (WCGNA) ${ }^{34}$ demonstrated a strong relationship between KDM4A, PAF1, and the KDM4A-9 and LSC17 GESs across AML. This network possessed high topological overlap (topological overlap matrix $(\mathrm{TOM}) \geq 0.05)$ ) with KDM4A as a highly connected node (Fig. 8E) suggesting that KDM4A-PAF1 regulates the KDM4A-9/LSC17 network in AML.

\section{KDM4A has a distinct function to another KDM4 family member, KDM4C in AML}

Previously, Cheung et al. showed that KDM4C is required for MLLr-AML cell survival ${ }^{8}$, indicating an overlapping role of KDM4A and KDM4C in AML. However, forced-expression of wild-type human KDM4C failed to rescue the clonogenic activity of murine MLLAF9 AML cells transduced with $k d m 4 a$ KD virus (Figs. S6A and S6B), suggesting KDM4A has a distinct role from that of KDM4C. This is in line with previously reported data showing no increase of global H3K27me3 level upon pharmacological inhibition of KDM4C in MLLr-AML cells $^{8}$. Consistently, at the molecular level, KDM4A KD led to transcriptional changes distinct from $K D M 4 C \mathrm{KD}$ via GSEA comparison (Fig. S6C), further supporting a unique role for KDM4A compared to KDM4C in human AML. In particular, KDM4A KD has no significant impact on gene expression of two established targets of KDM4C, HOXA9, and MEIS1 in human MLLr-AML cells. These results are also validated by Q-PCR using shRNAs targeting HOXA9 as control (Fig. S6D). More importantly, $k d m 4 c \mathrm{KD}$ had no impact on PAF1 expression, nor its associated genes targeted by KDM4A including KDM4A9 and LSC17 GESs (Fig. S6E). Together, these data demonstrate an essential role of KDM4A in human AML.

\section{Discussion}

Previous reports indicate that the KDM4 family is required for normal hematopoiesis ${ }^{9,35}$, whilst loss of individual members is tolerated in normal cells ${ }^{35}$ highlighting the importance of identifying KDM4 family members that are essential for the survival of AML cells. Our data demonstrate KDM4A is unique; it is selectively required for AML cell survival, with no negative effect on normal hematopoiesis offering a therapeutic window. Lack of tractable enzymatic activities limits the potential of PAF1 or other subunits of the PAFc as therapeutic targets in cancer. Herein, we identify a novel $K D M 4 A$ $P A F 1$ signaling axis co-regulating oncogenic transcriptional networks in human AML, providing a way to eliminate leukemic cells with broad therapeutic applications. Our study provides a strong rationale for the further development of KDM4A inhibitors, presenting a promising strategy for novel epigenetic-based therapy in AML.

$K D M 4 A-9$ shows strong therapeutic implications comparable with $L S C 17^{17}$. A high $K D M 4 A-9$ score may reflect an important biological property of KDM4A in leukemogenesis. The function of the KDM4A-9 genes in leukemogenesis is unknown; except Tetraspanin (CD82) $)^{36,37}$ which plays an important role in AML. Corroborating recent findings ${ }^{5,6}$, our data suggest KDM4A regulates H3K9me3 to direct leukemogenesis. H3K9me3 has emerged as a key player in repressing lineage- 
A

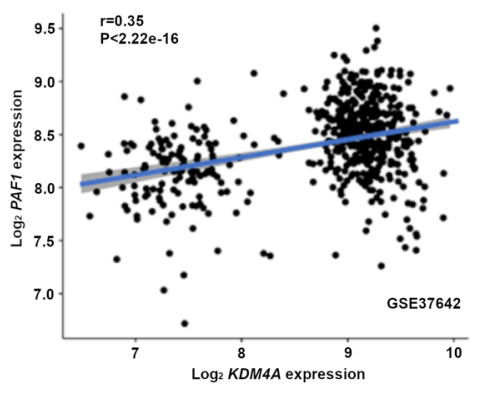

C

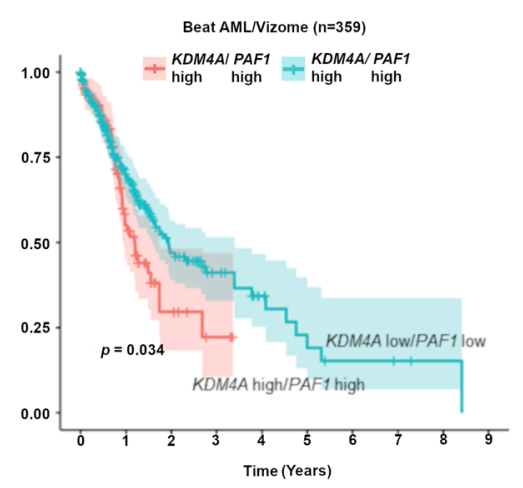

E

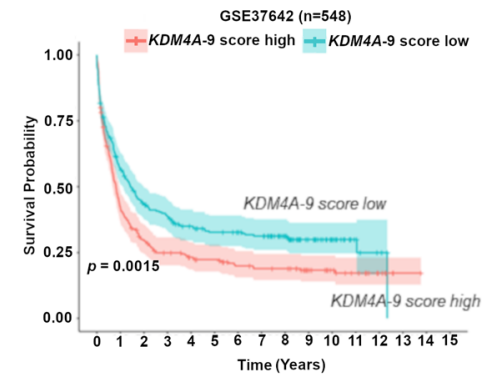

G

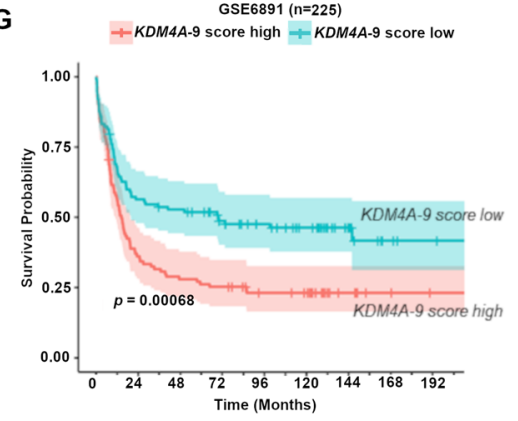

B
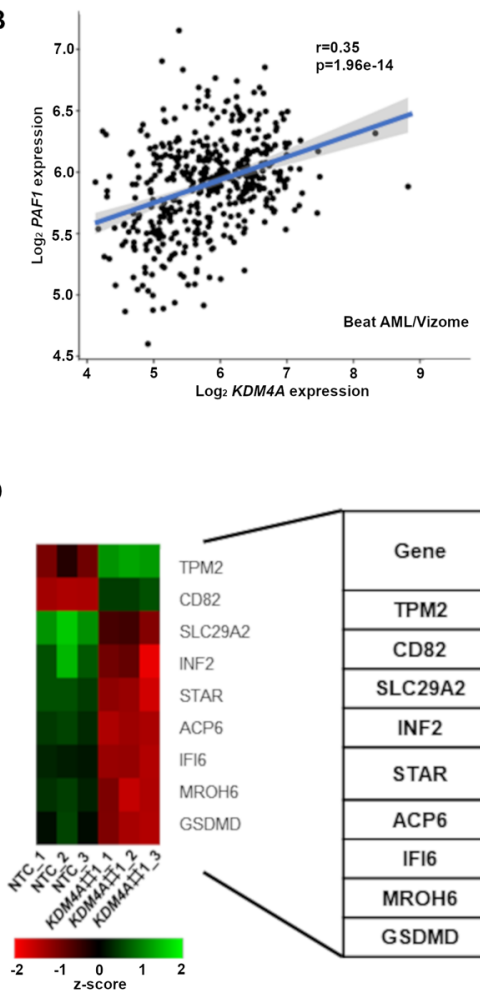

\begin{tabular}{l|c|c|c|}
\hline \multirow{2}{*}{$\begin{array}{l}\text { TPM2 } \\
\text { CD82 } \\
\text { SLC29A2 } \\
\text { INF2 } \\
\text { STAR } \\
\text { ACP6 } \\
\text { IFI6 }\end{array}$} & Gene & $\begin{array}{c}\text { RNA-seq } \\
\text { Log } 2 \text { FC }\end{array}$ & Coefficient \\
\cline { 2 - 4 } $\begin{array}{l}\text { MROH6 } \\
\text { GSDMD }\end{array}$ & TPM2 & 1.4408 & 0.0973 \\
\cline { 2 - 4 } & CD82 & 1.4062 & 0.0387 \\
\cline { 2 - 4 } & SLC29A2 & -1.0127 & 0.1675 \\
\cline { 2 - 4 } & INF2 & -1.0597 & 0.1500 \\
\cline { 2 - 4 } & STAR & -1.1859 & -0.1335 \\
\cline { 2 - 4 } & ACP6 & -1.2508 & 0.0463 \\
\cline { 2 - 4 } & IFI6 & -1.4429 & 0.0158 \\
\cline { 2 - 4 } & MROH6 & -1.4719 & 0.0867 \\
\cline { 2 - 4 } & GSDMD & -1.7703 & 0.0083 \\
\hline
\end{tabular}

$\mathbf{F}$

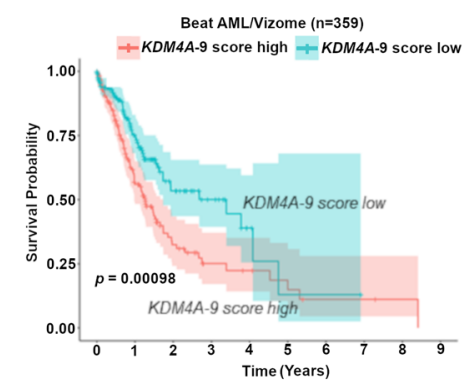

H

GSE12417 CN AML (n=160)

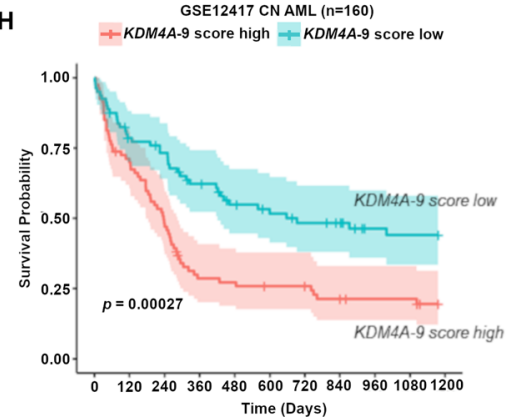

Fig. 6 A core 9-gene signature downstream of KDM4A strongly associated with clinical outcome. A-B Scatterplot showing the correlation between expression of KDM4A versus PAF1 in primary AML patient samples (GSE37642) (A) and (Beat AMLNizome), R by Pearson correlation, $p<0.05$ (B). C Kaplan-Meier survival analysis conducted in Beat AML dataset. Patients with both $K D M 4 A^{\text {high }}$ and $P A F 7^{\text {high }}$ expression have inferior overall survival. Patients dichotomized into high and low groups for KDM4A or PAF1 based on whether expression was above the median for each gene; $p$ by log-rank test. D Heatmap showing gene expression of the KDM4A-9 gene signature genes with the table of their respective regression coefficients and log 2 FC as determined by RNA-seq in KDM4A KD THP1 cells. E-H Kaplan-Meier survival analysis conducted in the large AML datasets (GSE37642) (E), (Beat AML/ Vizome) (F), (GSE6891) (G), and (GSE12417) (H) showing that the KDM4A-9 score can predict survival across AML patients of varying subtypes. Patients were dichotomized into high and low groups based on whether they possessed a score above or below the median signature score; $p$ by log-rank test. 


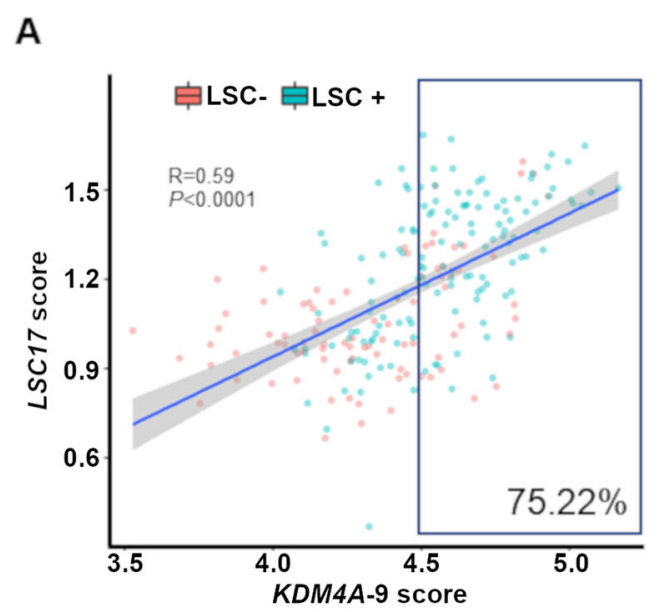

B

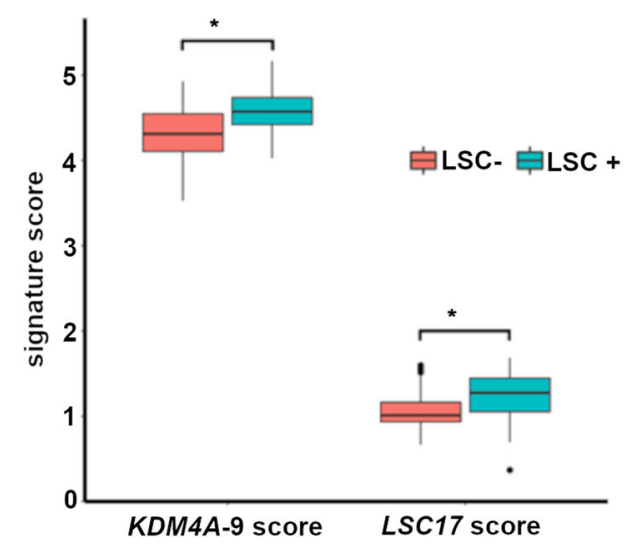

C
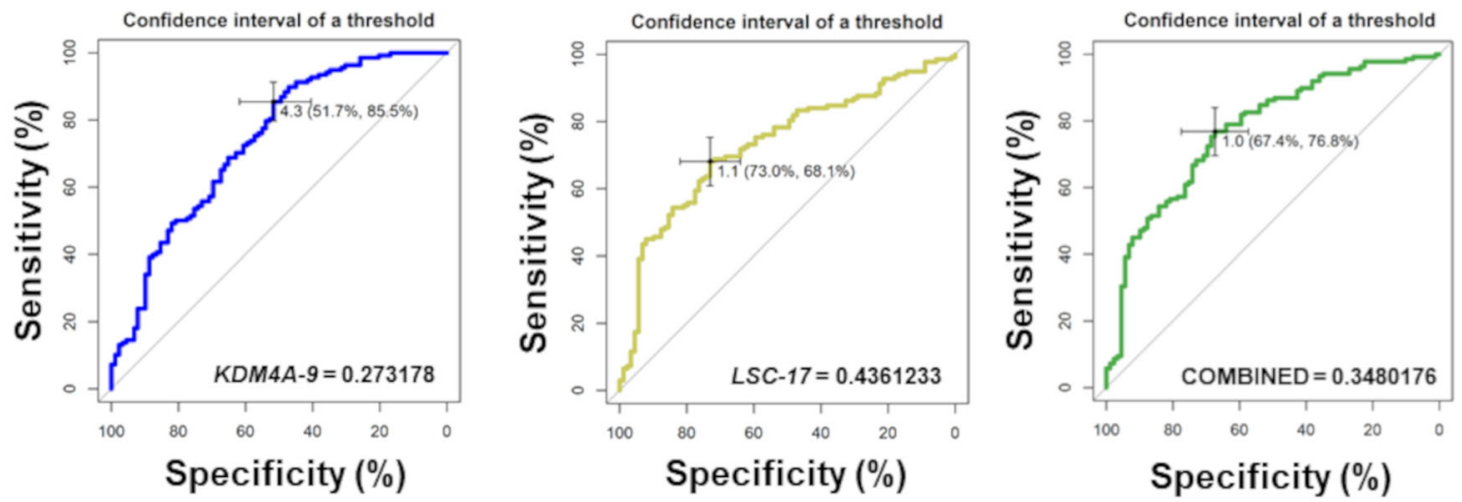

D

E
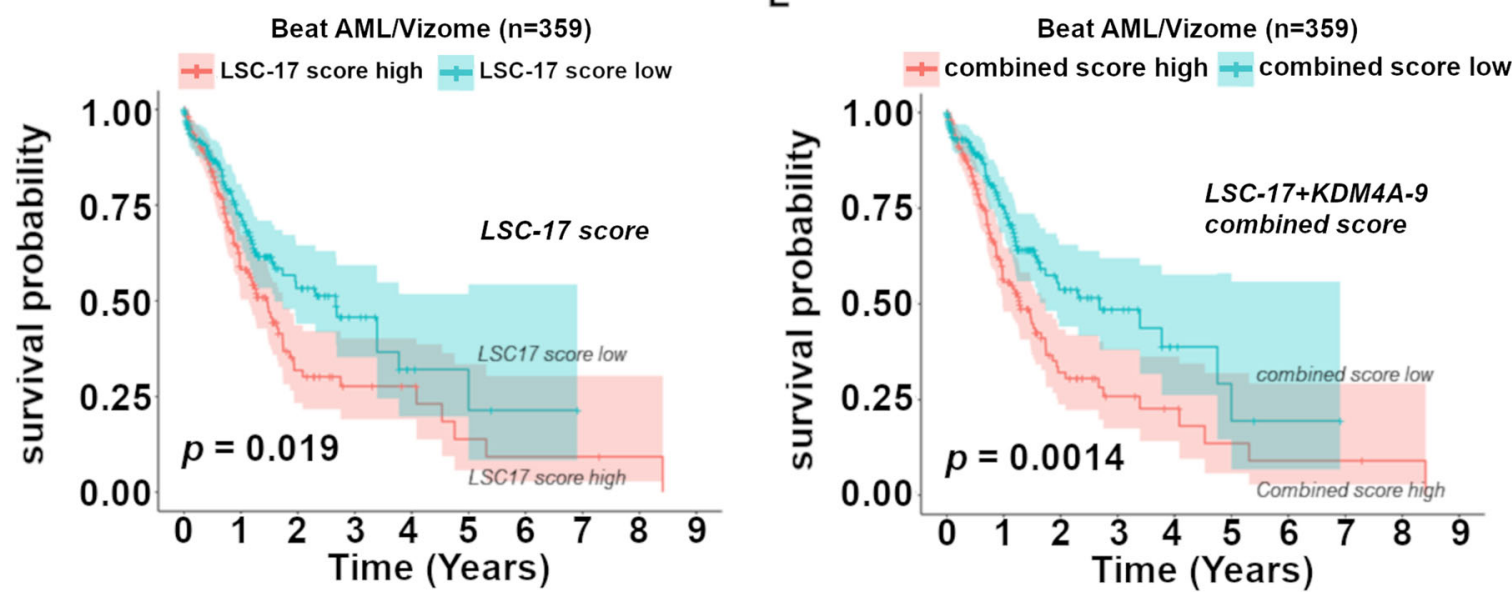

Fig. 7 KDM4A-9 enriched with LSC activity, is a poor prognosis marker for AML. A Scatterplot showing a moderate correlation between the KDM4A-9 score and LSC17 score in primary AML patient samples (GSE76008). LSC enriched (LSC,$+ n=138$ ) cell fractions from 78 patient samples are colored blue whilst those that lack LSC enrichment (LSC-, $n=89$ ) are colored red. Over 75\% of KDM4A-9 high score (above median value) fractions are LSC+. Pearson correlation used to assess correlation. Significance determined by $t$-test. B Box plot showing KDM4A-9 or LSC17 signature scores in two comparative groups: LSC+ and LSC - from (A); unpaired $t$-test, ${ }^{*} p<0.0001$. C ROC curves of KDM4A-9 (blue), LSC17 (yellow), and KDM4A-9/LSC17 (green) show the diagnostic capability of each signature to predict LSC enrichment in AML samples. The black bars in each plot are the $95 \%$ confidence intervals for the optimal cut-off. The Youden index was used to determine the optimal cut-off for each signature. D-E Patients in the Beat AMLNizome dataset were dichotomized into high and low groups based on whether they possessed a score above or below the median signature score. Kaplan-Meier survival analysis conducted showing that the combined KDM4A-9/LSC17 score (E) is effective in the prediction of AML patient survival over the LSC17 score alone (D). 

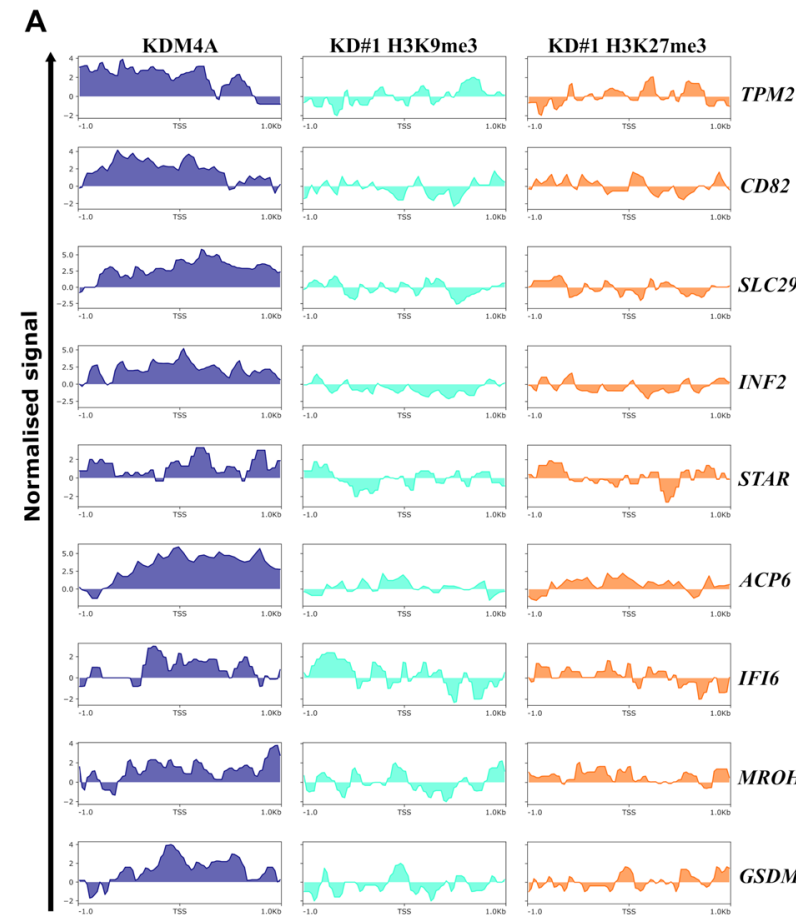

C
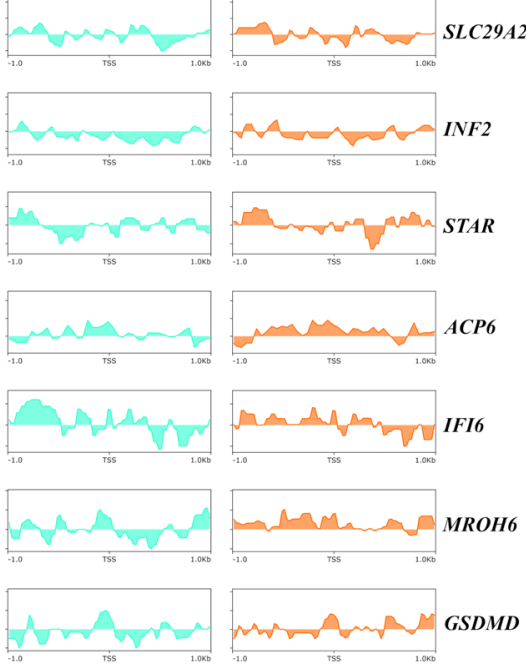

E
B

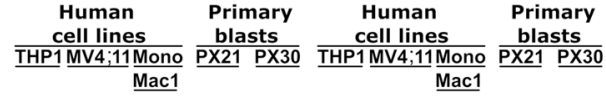

$t(9 ; 11) t(4 ; 11) t(9 ; 11) t(6 ; 11) t(10 ; 11) t(9 ; 11) t(4 ; 11) t(9 ; 11) t(6 ; 11) t(10 ; 11)$

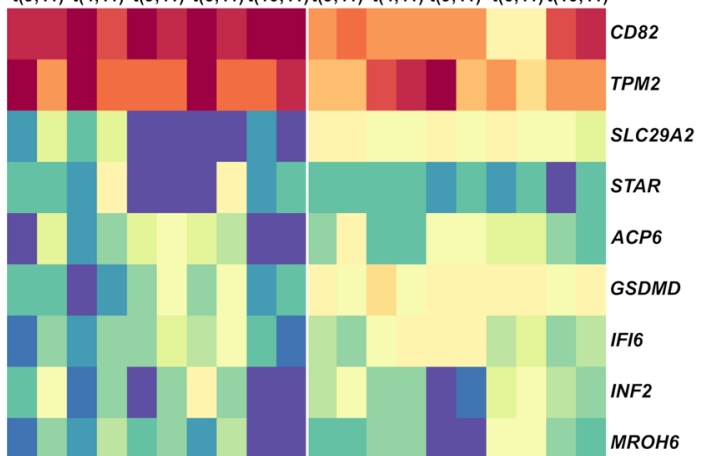

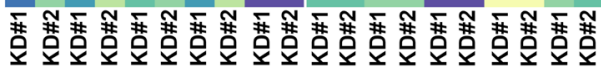

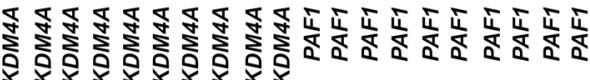

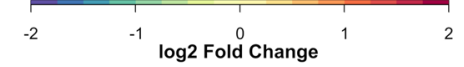

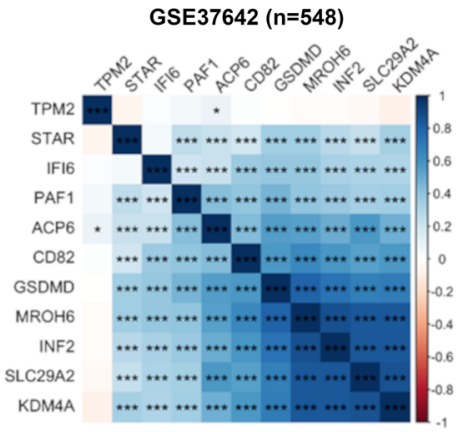

KDM4A9, LSC17 gene network in AML

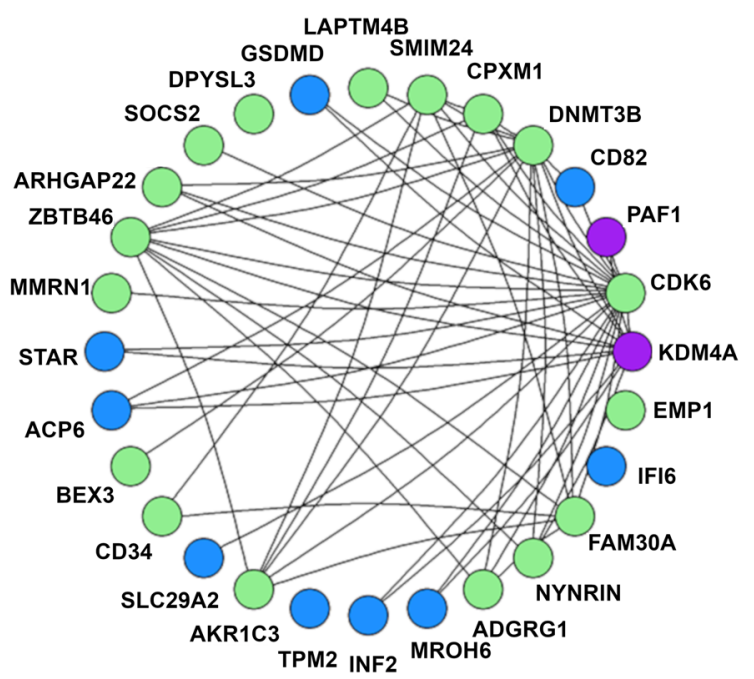

Beat AML/Vizome $(n=359)$

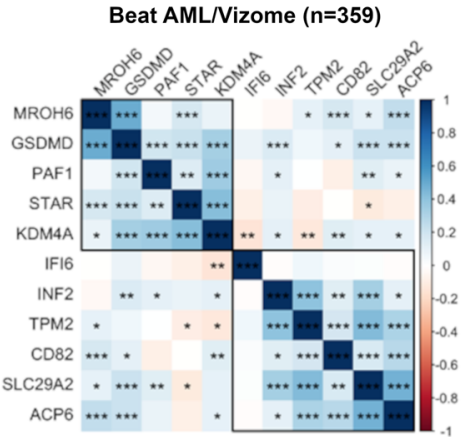

Fig. 8 KDM4A-mediated epigenomic network required for AML cell self-renewal and survival. A Heatmap showing relative expression of KDM4A-9 signature genes as determined by QPCR in the indicated human MLLr-AML cell lines and AML primary cells following KDM4A KD or PAF1 KD in comparison with NTC control cells $(n=3)$. B Input normalized ChIP-seq coverage tracks showing KDM4A ChIP signal in WT THP1 cells and H3K9me3/H3K27me3 ChIP signal normalized to NTC in KDM4A KD THP1 cells at KDM4A-9 signature genomic loci (+/-1 kb TSS). The normalized signal shown is the $\log _{2}$ ratio of read counts compared against input control. C-D Correlation matrices showing the Pearson correlation coefficients for KDM4A, KDM4A-9 genes, and PAF1 gene expression in GSE37642 (C) and Beat AML/Nizome (D) AML datasets. Significance determined by $t$-test; ${ }^{*} p$ $<0.05,{ }^{* *} p<0.01,{ }^{* *} p<0.001,{ }^{* * *} p<0.0001$. E KDM4A, PAF1, KDM4A-9 (in blue), and LSC17 (in green) gene network showing the topological overlap between genes as detected from $262 \mathrm{AML}$ samples (Beat AML) (the corresponding topological overlap matrix (TOM) $\geq 0.05$ between nodes. Genes with high topological overlap matrix ( $(\mathrm{OM})$ measure ( $\mathrm{TOM} \geq 0.05)$ have related functions). 
inappropriate genes, impeding the reprogramming of cell identity during development and cell fate determina$\operatorname{tion}^{24,38}$. It would be interesting to determine further the clinical diagnostic relevance of $\mathrm{H} 3 \mathrm{~K} 9 \mathrm{me} 3$ in relation to KDM4A in AML patients.

\section{Acknowledgements}

We thank Jennifer Cassels and Karen Dunn in Paul O'Gorman Leukaemia Research Centre, Glasgow, Gary Spencer, Jeff Barry, Abi Johnson, and staff from the Biological Resources Unit in CRUK Manchester Institute, for technical assistance. We thank Dr. Tim Somervaille for feedbacks and critical comments on the manuscript. We thank Dr. Peter J. M. Valk (Department of Hematology, Erasmus University Medical Centre, Rotterdam) for kindly providing the survival data for the GSE6891 data set and Dr. Tobias Herold and the AMLCG group for granting access to the clinical data for GSE37642.

\section{Author details}

'Paul O'Gorman Leukaemia Research Centre, Institute of Cancer Sciences, University of Glasgow, Glasgow, United Kingdom. ²Medicinal Chemistry Department, Joseph Black Building, School of Chemistry, University of Glasgow, Glasgow, United Kingdom. ${ }^{3}$ Cellular Signalling Unit, Department of Biomedical, Metabolic and Neural Sciences, University of Modena and Reggio Emilia, Modena 41125, Italy. ${ }^{4}$ Wolfson Wohl Cancer Research Centre, Institute of Cancer Sciences, University of Glasgow, Glasgow, United Kingdom

\section{Author contributions}

X.H. and M.E.M. designed the study. X.H., M.E.M., L.M., S.P., N.M., R.P.B., and A.H. performed the experiments. M.M. and X.H. analyzed genomic data and performed the statistical analysis. X.H. and M.M. wrote the manuscript. S.M., R.M.J.L., H.G.J., D.V., A.M.M., and X.H. provided critical support and supervised the study. All authors read and approved the manuscript.

\section{Funding}

This work was supported by the Wellcome Trust [105614/Z/14/Z]; Leuka [2016/ JGF/0005]; the Howat Foundation and Friends of Paul O'Gorman; Chief Scientific Officer (CSO) [CGA/19/63]; MRC CiC [18048]; Tenovus Scotland [S1913]. and SULSA-PECRE; X.H. is a John Goldman Fellow; M.M. is a recipient of Medical Research Council (MRC), UK DTP Ph.D. studentship [1732424]; L.M. is a recipient of Adam Renwick Martin-Friends of Paul O'Gorman Ph.D. Studentship; S.P. is a recipient of Carnegie Trust Ph.D. Studentship [PHD007721].

\section{Ethics approval and consent to participate}

The use of human tissue was in compliance with the ethical and legal framework of the United Kingdom's Human Tissue Act (2004) and the Human Tissue (Scotland) Act (2006). Normal CD34 ${ }^{+}$mobilized HSPC surplus to requirements were from patients undergoing chemotherapy and autologous transplantation for lymphoma and myeloma. Their use was authorized by the Salford and Trafford Research Ethics Committee and, for samples collected since 2006, following the written informed consent of donors. Normal human BM was collected with informed consent from healthy adult male donors, with the ethical approval of the Yorkshire Independent Research Ethics Committee. Primary human AML samples were from Manchester Cancer Research Centre's Tissue Biobank (instituted with approval of the South Manchester Research Ethics Committee) and Paul O'Gorman Leukaemia Research Centre's hematological cell research biobank (with approval of the West of Scotland Research Ethics Committee 4). Their use was authorized following project review by the Research Tissue Biobank's scientific sub-committee, and with the informed consent of donors.

\section{Conflict of interest}

The authors declare no competing interests.

\section{Publisher's note}

Springer Nature remains neutral with regard to jurisdictional claims in published maps and institutional affiliations.
Supplementary information The online version contains supplementary material available at https://doi.org/10.1038/s41419-021-03738-0.

Received: 10 October 2020 Revised: 2 April 2021 Accepted: 6 April 2021 Published online: 03 June 2021

\section{References}

1. Papaemmanuil, E. et al. Genomic classification and prognosis in acute myeloid leukemia. N. Engl. J. Med. 374, 2209-2221 (2016).

2. Serio, J. et al. The PAF complex regulation of Prmt5 facilitates the progression and maintenance of MLL fusion leukemia. Oncogene 37, 450-460 (2018).

3. Muntean, A. G. et al. The PAF complex synergizes with MLL fusion proteins at HOX loci to promote leukemogenesis. Cancer Cell 17, 609-621 (2010).

4. Chaudhary, K., Deb, S., Moniaux, N., Ponnusamy, M. P. \& Batra, S. K. Human RNA polymerase II-associated factor complex: dysregulation in cancer. Oncogene 26, 7499-7507 (2007)

5. Ropa, J. et al. PAF1 complex interactions with SETDB1 mediate promoter H3K9 methylation and transcriptional repression of Hoxa9 and Meis1 in acute myeloid leukemia. Oncotarget 9, 22123-22136 (2018).

6. Ropa, J. et al. SETDB1 mediated histone H3 lysine 9 methylation suppresses MLL-fusion target expression and leukemic transformation.Haematologica 105, 2273-2285 (2019).

7. Muller-Tidow, C. et al. Profiling of histone H3 lysine 9 trimethylation levels predicts transcription factor activity and survival in acute myeloid leukemia. Blood 116, 3564-3571 (2010).

8. Cheung, N. et al. Targeting Aberrant epigenetic networks mediated by PRMT1 and KDM4C in acute myeloid leukemia. Cancer Cell 29, 32-48 (2016).

9. Agger, K. et al. Jmjd2/Kdm4 demethylases are required for expression of II3ra and survival of acute myeloid leukemia cells. Genes Dev. 30, 1278-1288 (2016).

10. Huang, $X$. et al. Enhancers of Polycomb EPC1 and EPC2 sustain the oncogenic potential of MLL leukemia stem cells. Leukemia 28, 1081-1091 (2014).

11. Berry, W. L., Shin, S., Lightfoot, S. A. \& Janknecht, R. Oncogenic features of the JMJD2A histone demethylase in breast cancer. Int. J. Oncol. 41, 1701-1706 (2012).

12. Black, J. C. et al. KDM4A lysine demethylase induces site-specific copy gain and rereplication of regions amplified in tumors. Cell 154, 541-555 (2013).

13. Harris, W. J. et al. The histone demethylase KDM1A sustains the oncogenic potential of MLL-AF9 leukemia stem cells. Cancer Cell 21, 473-487 (2012).

14. Somervaille, T. C. \& Cleary, M. L. Identification and characterization of leukemia stem cells in murine MLL-AF9 acute myeloid leukemia. Cancer Cell 10 257-268 (2006).

15. Bray, N. L., Pimentel, H., Melsted, P. \& Pachter, L. Near-optimal probabilistic RNA-seq quantification. Nat. Biotechnol. 34, 525-527 (2016).

16. Friedman, J., Hastie, T. \& Tibshirani, R. Regularization paths for generalized linear models via coordinate descent. J. Stat. Softw. 33, 1-22 (2010).

17. $\mathrm{Ng}, \mathrm{S} . \mathrm{W}$. et al. A 17-gene stemness score for rapid determination of risk in acute leukaemia. Nature 540, 433-437 (2016).

18. Ravasz, E., Somera, A. L., Mongru, D. A., Oltvai, Z. N. \& Barabasi, A. L. Hierarchical organization of modularity in metabolic networks. Science 297, 1551-1555 (2002).

19. Chung, Y. G. et al. Histone demethylase expression enhances human somatic cell nuclear transfer efficiency and promotes derivation of pluripotent stem cells. Cell Stem Cell 17, 758-766 (2015).

20. Whetstine, J. R. et al. Reversal of histone lysine trimethylation by the JMJD2 family of histone demethylases. Cell 125, 467-481 (2006).

21. King, O. N. et al. Quantitative high-throughput screening identifies 8hydroxyquinolines as cell-active histone demethylase inhibitors. PLOS ONE 5, e15535 (2010).

22. Schiller, R. et al. A cell-permeable ester derivative of the JmjC histone demethylase inhibitor IOX1. ChemMedChem 9, 566-571 (2014).

23. Bavetsias, V. et al. 8-Substituted Pyrido[3,4-d]pyrimidin-4(3H)-one derivatives as potent, cell permeable, KDM4 (JMJD2) and KDM5 (JARID1) histone lysine demethylase inhibitors. J. Med. Chem. 59, 1388-1409 (2016).

24. Becker, J. S., Nicetto, D. \& Zaret, K. S. H3K9me3-dependent heterochromatin: barrier to cell fate changes. Trends Genet. 32, 29-41 (2016).

25. Morey, L. \& Helin, K. Polycomb group protein-mediated repression of transcription. Trends Biochem. Sci. 35, 323-332 (2010). 
26. $\mathrm{Yu}, \mathrm{M}$. et al. RNA polymerase $\|$-associated factor 1 regulates the release and phosphorylation of paused RNA polymerase II. Science 350, 1383-1386 (2015)

27. Kim, J., Guermah, M. \& Roeder, R. G. The human PAF1 complex acts in chromatin transcription elongation both independently and cooperatively with SII/TFIIS. Cell 140, 491-503 (2010).

28. Chen, F. X. et al. PAF1, a molecular regulator of promoter-proximal pausing by RNA polymerase II. Cell 162, 1003-1015 (2015).

29. Prange, K. H. M. et al. MLL-AF9 and MLL-AF4 oncofusion proteins bind a distinct enhancer repertoire and target the RUNX1 program in 11q23 acute myeloid leukemia. Oncogene 36, 3346-3356 (2017).

30. Zuber, J. et al. RNAi screen identifies Brd4 as a therapeutic target in acute myeloid leukaemia. Nature 478, 524-528 (2011).

31. Faber, J. et al. HOXA9 is required for survival in human MLL-rearranged acute leukemias. Blood 113, 2375-2385 (2009).

32. Ivanova, N. B. et al. A stem cell molecular signature. Science 298, 601-604 (2002).
33. Simon, N., Friedman, J., Hastie, T. \& Tibshirani, R. Regularization paths for Cox's proportional hazards model via coordinate descent. J. Stat. Softw. 39, 1-13 (2011).

34. Langfelder, P. \& Horvath, S. WGCNA: an R package for weighted correlation network analysis. BMC Bioinforma. 9, 559 (2008).

35. Agger, $\mathrm{K}$. et al. The KDM4/JMJD2 histone demethylases are required for hematopoietic stem cell maintenance. Blood 134, 1154-1158 (2019).

36. Marjon, K. D. et al. Tetraspanin CD82 regulates bone marrow homing of acute myeloid leukemia by modulating the molecular organization of $\mathrm{N}$-cadherin. Oncogene 35, 4132-4140 (2016).

37. Nishioka, C. et al. The novel function of CD82 and its impact on BCL2L12 via AKT/STAT5 signal pathway in acute myelogenous leukemia cells. Leukemia $\mathbf{2 9}$ 2296-2306 (2015).

38. Monaghan, L. et al. The emerging role of H3K9me3 as a potential therapeutic target in acute myeloid leukaemia. Front. Oncol. 9, 705 (2019). 